\title{
Philosophiques
}

\section{L’écriture de Nietzsche dans Zarathoustra}

\section{Serge Botet}

Volume 38, numéro 2, automne 2011

URI : https://id.erudit.org/iderudit/1007457ar

DOI : https://doi.org/10.7202/1007457ar

Aller au sommaire du numéro

\section{Éditeur(s)}

Société de philosophie du Québec

\section{ISSN}

0316-2923 (imprimé)

1492-1391 (numérique)

Découvrir la revue

Citer cet article

Botet, S. (2011). L'écriture de Nietzsche dans Zarathoustra. Philosophiques, 38(2), 383-417. https://doi.org/10.7202/1007457ar

\section{Résumé de l'article}

La Zarathoustra de Nietzsche, de son propre aveu l'opus magnum de Nietzsche a toujours été appréhendé sous l'angle de ses contenus et de ses thématiques : volonté de puissance, surhumain, éternel retour. Le vitalisme de Nietzsche, illustré par ces trois enseignements centraux de Zarathoustra, a rarement été recherché dans la forme et les caractéristiques précises d'un discours qui se voulait pourtant novateur et que l'on pouvait supposer - à l'opposé du discours neutre et reproducteur de la tradition philosophique - chercher à être lui-même " vital ». Pour les mêmes raisons, comment a-t-on pu négliger à ce point l'impact communicationnel qu'un tel discours se devait de produire sur ses lecteurs, à l'image du discours prosélyte que Zarathoustra adresse sans cesse à ses auditoires ? Si Zarathoustra est une apologie du vouloir exacerbé sous toutes ses formes, ne fallait-il pas justement qu'il fût un appel à vouloir, un manifeste plutôt qu'un traité. C'est ce renouveau qu'apporte le discours nietzschéen dans le discours philosophique séculaire, c'est sa fonction appellative que nous entendons analyser ici à l'aide d'une panoplie d'outils linguistiques allant de la morphosyntaxe aux modèles actantiels, mais toujours dans l'objectif de replacer ces caractéristiques dans une perspective philosophique.
Ce document est protégé par la loi sur le droit d'auteur. L’utilisation des services d'Érudit (y compris la reproduction) est assujettie à sa politique d'utilisation que vous pouvez consulter en ligne.

https://apropos.erudit.org/fr/usagers/politique-dutilisation/ 


\title{
L'écriture de Nietzsche dans Zarathoustra
}

\author{
SERGE BOTET \\ Université de Clermont-Ferrand 2
}

\begin{abstract}
RÉSUMÉ. - La Zarathoustra de Nietzsche, de son propre aveu l'opus magnum de Nietzsche a toujours été appréhendé sous l'angle de ses contenus et de ses thématiques: volonté de puissance, surhumain, éternel retour. Le vitalisme de Nietzsche, illustré par ces trois enseignements centraux de Zarathoustra, a rarement été recherché dans la forme et les caractéristiques précises d'un discours qui se voulait pourtant novateur et que l'on pouvait supposer - à l'opposé du discours neutre et reproducteur de la tradition philosophique chercher à être lui-même «vital». Pour les mêmes raisons, comment a-t-on pu négliger à ce point l'impact communicationnel qu'un tel discours se devait de produire sur ses lecteurs, à l'image du discours prosélyte que Zarathoustra adresse sans cesse à ses auditoires? Si Zarathoustra est une apologie du vouloir exacerbé sous toutes ses formes, ne fallait-il pas justement qu'il fût un appel à vouloir, un manifeste plutôt qu'un traité. C'est ce renouveau qu'apporte le discours nietzschéen dans le discours philosophique séculaire, c'est sa fonction appellative que nous entendons analyser ici à l'aide d'une panoplie d'outils linguistiques allant de la morphosyntaxe aux modèles actantiels, mais toujours dans l'objectif de replacer ces caractéristiques dans une perspective philosophique.
\end{abstract}

ABSTRACT. - Nietzsche's Zarathustra, the philosophical work that the philosopher regarded as his magnum opus, has always been approached through its contents and themes: the will to power, the Übermensch, Overman, eternal recurrence. Nietzsche's "vitalism," as illustrated by Zarathustra's three central teachings, has rarely been sought in the form and precise characteristics of a discourse that was intended to be innovative and could therefore itself be expected to be "vital," (in contrast with the neutral discourses that simply reproduced the philosophical tradition). Allowing this hypothesis, we must ask how interpreters could ignoreFor the same reasons, how could interpreters ignore to such an extent the communicative impact that such a discourse was intended to produce on its readers, following in its way the proselytizing discourse that Zarathustra restlessly addressed to his audiences? If Zarathustra represents the apologia of an exacerbated will in all its forms, is it not logical to regard Nietzsche's work as an appeal intended to strengthen that will; as a manifesto rather than a treatise? It is thus the renewal that Nietzsche's discourse brings to traditional philosophical discourse andthe appellative function it fulfills that we will analyse here, using a variety of linguistic tools ranging from morphosyntax to actantial models. Our aim will invariably be to set these features in a philosophical perspective and framework.

MOTS-CLÉS: appel, déconstruction, déconstructivisme, diégèse, interprétation, discours, métaphore, perspectivisme, récit, volonté de puissance. 


\section{Zarathoustra comme renouveau du discours philosophique}

Avant d'exposer les principales thèses qui justifient ce que suggère le titre de la présente étude, nous proposerons certains préalables méthodologiques sans lesquels notre lecture de l'œuvre, qui se veut novatrice, serait difficilement compréhensible. D'une façon générale, notre travail porte sur l'écriture philosophique au sens large, plus spécialement en tant que cette écriture est le support de signifiances philosophiques ${ }^{1}$. Autrement dit, notre objectif n'est pas simplement l'étude du style des philosophes sans lien avec leurs doctrines, mais l'étude de l'articulation de ces dernières dans des types spécifiques d'écritures qui, loin d'être accessoires, sont constitutifs de ces doctrines. L'objectif est en somme l'étude conjointe du «fond » et de la «forme » du discours philosophique, sachant que cette «forme», si elle est largement prise en compte dans l'étude du texte littéraire, est le plus souvent considérée comme assez accessoire en philosophie. L'opposition classique entre forme et fond est déjà en soi suffisamment parlante à ce propos: le fond est essentiel; la forme est plus ou moins accidentelle. Cela est sans doute plus vrai encore en philosophie. C'est ici l'idée qui prime; l'écriture, elle, exprime. Son rôle est fatalement second, ou du moins considéré comme tel. L'existence même de ce volet de la philosophie qualifié d' «histoire des idées" en est la preuve; il est rarement question de l'écriture ou de la formulation de ces idées. Bref, le «dire» de la philosophie est traditionnellement une sorte de point aveugle.

Bien sûr, nous n'entendons cela qu'en termes de tendances. Nombreux sont les philosophes pour lesquels doctrine et formulation furent indissociablement liées. Citons par exemple Descartes, dont la "Méthode " est impensable sans le récit de vie autobiographique qui en souligne les étapes ${ }^{2}$. Comme on l'a également montré, le même Descartes utilise dans ses Méditations la pluralité référentielle du déictique "je » pour construire au fil du texte - et faire participer didactiquement le lecteur à cette lecture du texte - un «je " générique qui constitue lui-même par étapes l'universalité du «je pense $»^{3}$. Finalement Descartes n'a-t-il pas (est-ce un hasard?) exposé des thèses sensiblement similaires de deux façons différentes dans le Discours et dans les Méditations? Dans ce registre, on pourra également citer l'exposé more geometrico de Spinoza dans son Éthique. Comme l'énonce Frédéric Cossuta, l'exposition géométrique retenue par Spinoza (divisions et subdivisions en paragraphes renvoyant les uns aux autres de

1. Nous reviendrons sur cette notion.

2. Frédéric Cossuta, "Argumentation, ordre des raisons et mode d'exposition dans l'œuvre cartésienne ", Descartes et l'argumentation philosophique, Paris, PUF, I996, p. I I I-I 85.

3. Magid Ali Bouacha écrit en conclusion de l'analyse qu'il fait de ce phénomène: «Les méditations peuvent ainsi se lire comme le lieu discursif où se joue la transformation de la première personne, passant du simple marquage indiciel au rang de catégorie conceptuelle » ( "De l'Ego à la classe des locuteurs: lecture linguistique des Méditations", in L'analyse du discours philosophique, Langages n ${ }^{\circ}$ I 9 , Paris, Larousse, septembre I995, p. 79-94). 
façon synoptique) permet plusieurs lectures échelonnées qui se réfèrent étroitement à des moments précis de la doctrine débouchant sur le vrai ${ }^{4}$. L'une et l'autre doctrine gèrent respectivement leur «forme » en fonction de leur teneur philosophique, à savoir la transcendance du sujet pensant chez Descartes, l'immanentisme de la substance chez Spinoza.

Mais à notre sens, ces isomorphismes entre fond et forme, si usuels et significatifs fussent-ils, ne remettent pas foncièrement en question le primat de l'idée sur l'expression qui marque l'histoire des idées. Même si l'on ne peut dénier aux philosophes susmentionnés un degré parfois significatif de "conscience» des formes, il nous semble que c'est à une philosophie plus tardive que revient le mérite d'avoir porté un véritable regard spéculaire sur sa propre activité consistant à philosopher et à écrire de la philosophie. Cette orientation fut d'ailleurs contemporaine d'un certain scepticisme ambiant vis-à-vis de la langue et du langage, dont on interrogea de plus en plus les limites, tout en exploitant leurs ressources suggestives. On ne peut rien dire de vraiment nouveau avec des mots usés, et surtout, on ne peut parler de l'être et laisser de côté le dire de l'être. Des philosophes comme Heidegger ou Nietzsche eurent, à notre avis, une conscience aiguë de cette aporie qui les mit pour la première fois en rupture radicale avec la tradition du primat de l'idée.

L'un comme l'autre auraient certainement voulu se passer du langage, le premier pour manifester la différence être/étant, l'ouverture de l'étant à son être, le second pour manifester la volonté de puissance. Mais comme solution de remplacement à la parole, il n'y a que le silence, et le silence est synonyme de vide philosophique. Si langue et langage sont donc un passage forcé, ils sont interrogés et explorés par le philosophe avec une acuité d'autant plus grande qu'ils sont considérés comme des moyens inadéquats; aux limites du dicible et du communicable, la langue et le langage, particulièrement ceux que la tradition philosophique a forgés, sont des obstacles. L'objectif du philosophe sera donc de les "pervertir», de les détourner de leurs fonctions, mais aussi d'exploiter corrélativement les ressources insoupçonnées qu'ils recèlent. C'est ainsi que nous avons proposé - il y a déjà quelques années - l'hypothèse que Heidegger manipulait la syntaxe de la langue allemande ${ }^{5}$ pour lui faire "dire» au plus près la différence ontologique, la différence de l'être et de l'étant, et c'est ainsi également que nous en sommes

4. «Le procès de lecture est donc en même temps un élargissement des horizons de la pensée vers l'universalisation du vrai $[. .$.$] pour que cet «ensemble» ne soit plus une simple$ virtualité, mais devienne un fait, il faut franchir une dernière étape: la lecture achevée est celle qui pourrait abolir la nécessité de la lecture, tellement l'entendement s'identifierait à l'essence manifestée par la forme du contenu. Lire c'est donc devenir en acte ce qu'on est en puissance, c'est-à-dire devenir Dieu (Ibid., p. 37).

5. Serge Botet, Langue, langage et stratégies linguistiques chez Heidegger, Bern, Peter Lang, I997, p. 25 I-265: La "dif-férence» (Unter-Schied), scindée par un trait d'union-désunion, est bien cette différence qui ne se «fixe» jamais et se maintient dans l'ouverture. 
arrivé, dans des travaux plus récents, à formuler l'hypothèse que Nietzsche mobilisait - non pas tant la langue et ses structures - mais plutôt cette fois le potentiel dynamique du discours, aspirant ainsi à dynamiser sa philosophie, à rendre en quelque sorte sa philosophie de la vie elle-même plus "vivante». Nietzsche, le "généalogiste", proclamateur d'une sorte de panvitalisme régi de part en part par la volonté de puissance, était à nos yeux susceptible de considérer également sa propre philosophie comme un «acte vital».

Nietzsche avait-il - comme Heidegger mais avec des objectifs sensiblement différents - tenté de transmettre ce qui est pour lui fondamental, à savoir la "vie» ou "volonté de puissance", autrement qu'au moyen de concepts, à savoir au moyen de signifiances ${ }^{6}$ données à décrypter au lecteur, ces signifiances étant en l'occurrence des signifiances « discursives ", relevant de la dynamique de la production/formulation/communication du message au sens le plus large?

\section{Questions de méthode}

Notre méthode d'analyse axée sur le texte, sur ses structures et sur les processus discursifs qui le sous-tendent étant assez spécifique et peu habituelle en philosophie, cette méthode faisant également partie intégrante de notre approche philosophique de Nietzsche au point d'être quasiment consubstantielle à nos thèses, nous nous permettrons d'en décrire assez précisément les lignes de force. Les spécificités méthodiques propres à chacune des deux grandes études que nous évoquons dans ce travail seront mentionnées au fil du texte.

\subsection{La notion de «signifiance»}

Dans son livre L'homme de paroles, Claude Hagège parle de «signifiance» quand il ne s'agit ni de signification, ni de sens "phénomène propre au texte comme combinaison de signes dans une situation donnée de parole ${ }^{7}$, mais de phénomènes échappant au codage caractérisé par un consensus explicite. Les signifiances, à la différence des significations, ne dénotent pas une réalité extra-linguistique précise (concrète ou abstraite) qu'il est convenu de dénoter de cette façon dans telle ou telle communauté linguistique; elles ne font que connoter une telle réalité, la suggérant de manière détournée par le biais d'associations, aussi bien en ce qui concerne la production du message (encodage), qu'en ce qui concerne sa réception (décodage). Autrement dit, une signifiance ne désigne rien directement, elle suggère indirectement quelque chose par le biais de l'interprétation. Par corollaire, à la différence d'une signification, qui est un investissement sémantique relativement stable,

6. Nous revenons ci-dessous sur cette notion.

7. Claude Hagège, L'homme de paroles, contribution linguistique aux sciences humaines, Paris, Folio Essais, I98 5, p. 293. 
généralement supporté par un lexème répertorié, une signifiance peut s'investir dans toutes sortes d'éléments, de structures, ou même de configurations actantielles qui sont a priori dépourvues de sens, ce sens étant précisément surimposé à ces supports non signifiants en soi par l'interprétation. Dans notre optique consistant à chercher à dégager de l'œuvre de Nietzsche des signifiances philosophiques, tout le problème était de prévenir les dérives interprétatives et d'éviter de "plaquer» sur le texte un sens totalement arbitraire. Sans vouloir nous engager dans des considérations trop théoriques, nous faisons ici un bref rappel de la position de Umberto Eco, exposée dans son ouvrage Les limites de l'interprétation. Tout en soulignant la part inévitablement subjective que comporte toute interprétation, Eco développe l'idée qu'une interprétation est bonne (le moins arbitraire possible) lorsqu'elle est corrélative d'une économie interprétative raisonnable, c'est-à-dire lorsqu'elle est étayée par une quantité et une convergence suffisantes de régularités et de récurrences observées dans le texte même ${ }^{8}$. La construction de la signifiance, étayée sur le cumul et la convergence des informations non explicites fournies par le texte, doit au final permettre une lecture cohérente de ce texte sur fond de philosophie. Précisons enfin que, $\mathrm{du}$ fait les signifiances peuvent s'investir dans toutes sortes de supports textuels et discursifs (lexique, tropes, syntaxe, etc.), il était absolument indispensable de délimiter un domaine d'investigation. Nous avons interrogé dans le Zarathoustra les signifiances relevant du domaine discursif au sens large, c'est-à-dire de la dynamique de la production/formulation/ communication du message.

\subsection{Discours «fondateur » et discours «constituant»}

Une autre notion clé au plan de la méthode d'investigation est le concept de discours «fondateur»/discours «constituant» développé par Cossuta ${ }^{9}$ et Maingueneau ${ }^{10}$. Nous n'avons retenu qu'une partie des critères assez complexes proposés par les auteurs pour définir ces notions: nous qualifions de «fondatrices» les caractéristiques visant — de manière «externe » - à positionner le discours comme «archidiscours» dans l'ensemble de tous les discours possibles (interdiscours), à mettre en quelque sorte ce discours en surplomb de tous les autres. Quant aux caractéristiques du discours dites «constituantes", ce sont celles qui entretiennent des rapports «internes» avec la doctrine, à savoir que cette doctrine projette sa spécificité dans la manière dont elle est produite, formulée et communiquée.

8. Cf. Umberto Eco, Les limites de l'interprétation, traduit de l'italien par Myriam Bouzaher, Paris, Bernard Grasset, Bibio Essais, 1992.

9. Frédéric Cossuta, Eléments pour la lecture des textes philosophiques, Paris, Bordas, I989.

10. Dominique Maingueneau, «L'énonciation philosophique comme institution discursive ", in Langages $n^{\circ} 119$, L'analyse du discours philosophique, op. cit, p. 40-62. 
Pour illustrer cela, nous donnerons un seul exemple assez parlant, même s'il n'a a priori que peu de rapport avec Nietzsche: l'existentialisme. Une caractéristique "doctrinale » de l'existentialisme est que toute théorie est coextensive à l'existence prise comme totalité: l'essence se confond avec l'existence. Il n'est donc pas surprenant que la production et la communication du discours existentialiste fonctionnent, elles aussi, sur un mode totalisant; l'existentialisme investit toutes les sphères de l'existence; on philosophe aussi bien en écrivant des pièces de théâtre, des romans ou des traités, qu'en devenant journaliste ou acteur de la vie politique, ou encore en adoptant une attitude et un mode de vie existentialistes (fréquentation des cafés, etc.). L'existentialisme fonde sa légitimité dans l'interdiscours en investissant tous les autres discours, ce qui est finalement une façon de se positionner «en surplomb» de ces derniers. C'est en ce sens que nous parlons de discours fondateur. Quant à la formulation de ce même discours existentialiste, il est indéniable qu'elle entretient des rapports internes avec la doctrine. Toujours très schématiquement: c'est parce que l'existentialisme est une philosophie du sujet et de la liberté qu'il s'écrit si souvent à la première personne; "c'est ce sujet que j'ai à être", écrit Sartre à plusieurs reprises dans L'Être et le Néant ${ }^{11}$. Pourtant ce n'est pas de Sartre qu'il s'agit, mais d'un sujet universel. Simplement, ce sujet-là ne peut se dire qu'à la première personne, l'idée à exprimer impose un certain mode énonciatif. De même, c'est parce qu'il privilégie le vécu par rapport à la théorie que l'existentialisme s'expose sous forme de récit narrativisé, etc. Bref, la doctrine informe le discours, qui fait lui-même écho à la doctrine. C'est en ce sens que nous parlons de discours constituant.

Cossuta et Maingueneau énoncent donc en substance que la philosophie (bien qu'elle prétende souvent le faire) ne se fonde et ne se constitue pas seulement à travers les contenus explicites qu'elle expose, elle se fonde et elle se constitue aussi à travers des procédés discursifs a priori non signifiants ou non déclarés l'être, et dont se dégagent pourtant à l'analyse ce qui semble correspondre à ce que nous avons appelé (bien que Cossuta et Maingueneau n'utilisent pas le terme) des "signifiances", qui peuvent elles-mêmes être vecteurs de philosophèmes.

\subsection{Apories et solutions}

Cela dit, nous nous sommes rendu compte, en l'appliquant au Zarathoustra, à quel point cette notion de signifiance pouvait poser de graves problèmes sur le plan heuristique. La position de Cossuta et Maingueneau semble impliquer que tout discours philosophique se fonde et se constitue à travers une discursivité latente que, la plupart du temps, il ignore. C'est donc à son corps défendant, à son insu même, qu'un tel discours, radiographié par

11. L'être et le néant, essai d'ontologie phénoménologique, Paris, Gallimard, I943. 
l'analyse du discours philosophique, livre les «signifiances » qui permettent justement de l'identifier comme fondateur et constituant. Autrement dit, les signifiances peuvent fort bien n'apparaître qu'au décodage, sans avoir été délibérément encodées. Dans la terminologie de U. Eco, ces signifiances relèveraient donc non de l'intention de l'auteur, qualifiée d'intentio auctoris, mais plutôt d'une intentio lectoris qui viendrait s'ajouter à la première pour lui faire dire ce qu'elle ne veut pas dire nécessairement, ou encore d'une intentio operis ${ }^{12}$ postulant une sorte d'autarcie du message philosophique, la forme discursive dégageant de façon automatique et par elle-même (indépendamment de l'intention de l'auteur et du lecteur) des signifiances idoines aux contenus véhiculés. Sauf à considérer l'analyste du discours comme le véritable philosophe et prôner la suprématie (qu'il faudrait à son tour justifier!) d'une métaphilosophie sur la philosophie elle-même, la question se posait donc de savoir s'il fallait aussi considérer ces «signifiances non intentionnelles ", construites après coup, comme de véritables philosophèmes, puisqu'elles ne sont pas censées avoir été voulues au départ par le philosophe.

Disons-le clairement: notre objectif n'était pas celui des analystes du discours philosophique, du moins pas pour autant qu'ils entendent "supplémenter » le travail du philosophe. Au demeurant, comme le suggère la notion d'intentio operis de Eco, on ne peut exclure qu'une doctrine philosophique (forme du contenu) puisse dans une certaine mesure conditionner les modalités de sa production/formulation/communication (forme de l'expression) sans aucune intention délibérée de l'encodeur ou du décodeur. En ce sens, la position de l'analyse du discours philosophique par rapport à la philosophie elle-même devient plus «tenable »: il ne s'agit pas d'adopter vis-à-vis de la philosophie en général une attitude dominante et conflictuelle (métaphilosophie), mais d'éclairer ses zones d'ombre et ses non-dits, tout en restant en phase avec elle. Tel était précisément notre objectif.

Mais notre paradigme étant un paradigme philosophique où le philosophe est présumé plus ou moins "maître de son discours », notre hypothèse de départ était surtout que Nietzsche avait lui-même programmé les signifiances que l'analyste du discours (que nous étions nous-même en l'occurrence) pouvait déchiffrer dans ses écrits. Si la philosophie "classique» est "histoire des idées ", si elle est en quelque sorte aveugle à sa propre discursivité, si les signifiances que l'analyste du discours philosophique peut y décrypter relèvent donc la plupart du temps de l'intentio operis (ou dans le pire des cas de l'intentio lectoris), nous cherchions pour notre part à démontrer que les signifiances nietzschéennes relevaient, elles, pleinement de l'intentio auctoris. Avec le Zarathoustra de Nietzsche, le discours philosophique cessait justement d'être aveugle à lui-même, mieux: ce discours véhiculait des signifiances qui étaient des philosophèmes à part entière, peu ou peu précisément identifiés jusque-là. 
Dans la présente étude, nous sommes donc parti d'une idée inspirée de F. Cossuta ${ }^{13}$ et M. Maingueneau ${ }^{14}$, idée qu'ils ne développent pas dans leurs travaux, mais qui découle implicitement de leurs thèses, à savoir que la philosophie pourrait avoir cherché dès les origines à occulter sa propre discursivité pour se transformer en ce que nous concevons comme une sorte d' «épiphanie de la vérité ». Tendanciellement (ce n'est pas une règle absolue, cf. supra), la philosophie se réduit à ce qu'il est convenu d'appeler l'histoire des idées, occultant largement le fait qu'elle est aussi l'histoire du discours sur les idées. Par sa vocation totalisante, elle tend à évacuer du champ du réel ce qu'elle n'explique pas et elle fait a fortiori le silence sur les conditions qui la rendent elle-même possible. Pas de philosophie sans discours philosophique; certes, mais cela, la philosophie ne peut le dire, du moins si elle veut rester la philosophie que Platon (entre autres) a instituée comme genre: la recherche de la vérité comme adéquation entre l'idée et les choses.

Au regard des savoirs avalisés dont nous disposions sur Nietzsche (cf. supra), l'hypothèse que ce dernier ait pu chercher à réintroduire en philosophie cette discursivité historiquement occultée était largement pertinente. La production/formulation/communication du message philosophique, loin d'être un donné accessoire sans incidence sur la nature du message philosophique, pouvait influer sur ce message et même constituer l'essentiel de celui-ci, donc participer de sa "vérité ${ }^{15}$. C'est ce que nous avons cherché à vérifier dans ce travail.

\section{Signifiances métadiscursives}

\section{1 «Métadiscours 》 et «présupposé discursif»}

Concrètement, nous nous sommes proposé d'étudier ce que nous avons appelé le "métadiscours ", qui "environne » le Zarathoustra en amont et en aval. Comme chez Heidegger, qui annotait le «Hüttenexemplar» de son ouvrage Sein und Zeit de la désormais célèbre formule "Wege, nicht Werke", l'œuvre de Nietzsche semble (pour d'autres raisons) incapable de "contenir» une philosophie qui la «déborde» de toutes parts. À preuve par exemple les très nombreuses mentions $d u$ nom de "Zarathoustra ", œuvre ou personnage, éparpillées dans l'ensemble des écrits de Nietzsche, antérieurs ou postérieurs à l'œuvre elle-même, tant dans la correspondance que dans les publications. Le phénomène est trop flagrant pour être fortuit. On pense, entre autres, au très célèbre $\mathbb{S} 342$ du Gai savoir (Die fröhliche

13. Éléments pour la lecture des textes philosophiques, op. cit.

14. "L'énonciation philosophique comme institution discursive", in Langages $n^{\circ} 119$, L'analyse du discours philosophique, op. cit.

15. Josef Simon écrit à ce sujet - en liaison avec le Zarathoustra: «Die Erfindung des Zarathustra hat demnach mit der Kritik am metaphysischen Wahrheitsbegriff zu tun", Ein Text wie Nietzsches Zarathustra, in Volker Gerhardt (Hg.), Also sprach Zarathustra, Berlin, Akademie Verlag, Klassiker Auslegen, 2000, p. 24I. 
Wissenschaft) intitulé Incipit tragœedia (KGW V.2.25I), qui est — à très peu de choses près - une réplique (anticipée) du début du prologue de Zarathoustra. À ce propos, un auteur comme Wolfram Groddeck a d'ailleurs démontré que Nietzsche aurait cherché à créer un réseau "labyrinthique " liant le Zarathoustra au reste de ses écrits ${ }^{16}$. Ces très nombreuses évocations du nom de "Zarathoustra » autour de l'œuvre sont donc potentiellement le support d'une première signifiance; en marge du Zarathoustra proprement dit, un métadiscours satellite en conditionne la lecture, créant ce que nous appelons à la suite de Oswald Ducrot, un "présupposé discursif » ${ }^{17}$. "Zarathoustra" (héros ou titre) est d'emblée omniprésent dans l'ensemble de l'œuvre qu'il paraît avoir "habité de tout temps ». Le début du prologue de Zarathoustra, un début in medias res extrêmement abrupt ${ }^{18}$, est d'ailleurs assez révélateur à cet égard: "Als Zarathustra dreissig Jahr alt war, verliess er seine Heimat» (KGW VI.I.5). Point n'est besoin de présenter un personnage déjà universellement connu. L'entrée de Zarathoustra dans l'histoire est parfaitement impromptue; grâce au présupposé discursif, Nietzsche impose son héros, son œuvre, et donc son propre discours comme radicalement fondateur dans le champ interdiscursif.

Mais le présupposé discursif fait aussi sentir ses effets sur le plan de la constitution doctrinale (au sens de Cossuta et Maingueneau). Le fait que les mentions de "Zarathoustra» (œuvre ou personnage) irradient à ce point au-delà des limites de l'œuvre, suggère du même coup que la philosophie n'est pas un produit fini conditionné dans un contenant prédéfini, en l'occurrence l'œuvre individuelle, mais bien un procès en marche se constituant ad hoc dans la dynamique sans fin du discours. Gageons que si Nietzsche avait pu écrire d'autres œuvres, "Zarathoustra" aurait sans doute continué à vivre dans celles-ci; en termes de signifiances, on voit une philosophie du «devenir» se profiler derrière une philosophie séculaire de l' «être». Mais cela, Nietzsche ne peut se contenter de l'affirmer comme simple profession de foi théorique; il le pratique en quelque sorte, il le vit même, à travers sa façon d'écrire sa philosophie "en continu ", non pas dans, mais au travers des œuvres.

16. Wolfram Groddeck, "Die 'Neue Ausgabe' der 'Fröhlichen Wissenschaft", in Nietzsche-Studien, Internationales Jahrbuch für die Nietzsche-Forschung, Berlin, New York, Walter de Gruyter, Bd. 26, I997, p. I94.

17. Oswald Ducrot évoque notamment cette notion de "présupposé » dans son ouvrage Dire et ne pas dire, principes de sémantique linguistique. Pour Ducrot, «introduire dans le discours des présupposés » équivaut à rendre "prisonnier " " son interlocuteur en lui imposant un « devoir croire ": "La présupposition serait une affirmation "faite en passant", que le destinataire doit croire» (Paris, Hermann, Savoir, Sciences, I99I, p. 96).

18. Remarquons qu'un auteur tel que Hans-Martin Gauger voit dans ce qu'il appelle la "medias-in-res-Technik» une caractéristique récurrente de l'écriture de Nietzsche qu'il conçoit comme "Mittel der Verlebendigung ( "Nietzsches Stil am Beispiel von Ecce homo », Nietzsche Studien, internationales Jahrbuch für die Nietzsche-Forschung, op. cit., Bd I3, p. 344-345). 
De plus, le passage en force qu'implique l'usage du présupposé discursif est un phénomène passablement inhabituel, pour ne pas dire « déviant " en philosophie. Le plus souvent la philosophie prépare son lecteur, elle argumente et explique plus qu'elle n'impose et assène. Un tel passage en force a donc toutes les chances de n'être ni gratuit ni fortuit: il renvoie manifestement à une autre composante de la «doctrine» nietzschéenne, à savoir, la vie comme combat, la vie comme champ de forces antagonistes, etc. À propos de l'œuvre de Nietzsche, Gilles Deleuze écrit: «Toute force est en rapport avec d'autres, soit pour obéir, soit pour commander ${ }^{19}$. " La réalité s'épuise dans ce rapport entre des forces dominantes et des forces dominées. Et là non plus, il ne s'agit pas d'une simple posture théorique: tous les schémas actantiels du Zarathoustra corroborent ce phénomène; Zarathoustra ordonne, enjoint, conseille, bref, il cherche à "agir » sur son auditoire, à exercer sur lui sa domination (nous reviendrons sur ce point). Mais - et c'est là l'aspect novateur - Zarathoustra n'est pas le seul à ordonner et à imposer; par le biais du présupposé, c'est aussi Nietzsche qui, d'un même geste, impose son Zarathoustra dans l'interdiscours, mettant ainsi sa pratique à l'unisson de sa propre philosophie; la stratégie fondatrice se double d'une stratégie constituante. Depuis les marges métadiscursives de l'œuvre se construit une nouvelle fois une signifiance philosophique.

\subsection{Ecce homo}

Toujours dans le cadre du métadiscours qui «environne» le Zarathoustra, il fallait bien sûr tenir compte de l'auto-commentaire rétrospectif que constitue Ecce homo. Là aussi plusieurs signifiances convergentes suggèrent, de manière plus ou moins explicite, un discours qui se fonde en se positionnant «en surplomb» des autres dans l'interdiscours. Nietzsche évoque par exemple la "hauteur»: "6000 Fuss jenseits von Mensch und Zeit» (KGW VI.3.333), le caractère contraignant: "er überfiel mich» (KGW VI.3.335), ou encore le caractère de révélation: "Offenbarung» (KGW VI.3.337) de l'inspiration qui lui aurait dicté son Zarathoustra. Il n'est pas question en l'occurrence de biographie intellectuelle (rien à voir, par exemple, avec Descartes qui retrace la genèse de sa démarche dans son Discours de la méthode). Ici, l'inspiration ne s'explique pas, elle est, au même titre que la révélation chrétienne, un «don du ciel» (Offenbarung). C'est le thème du "fiat» verbal que nous avons eu l'occasion de développer en d'autres lieux: comme la parole du "Livre», la parole de Zarathoustra devient créatrice, capable de faire surgir un monde à partir de rien. Tout en prenant le contre-pied de la religion instituée, Nietzsche confère à sa philosophie la même hauteur sacrale; n'avait-il pas d'ailleurs qualifié lui-même son «Zarathoustra» de cinquième Évangile? Il est inutile d'épiloguer sur l'effet fondateur de ces phénomènes (en termes de signifiances, bien entendu).

19. Nietzsche et la philosophie, Paris, Quadrige/PUF, I962, p. 45. 
Mais ce n'est pas tout: ce dispositif fondateur a également des répercussions au plan de la constitution doctrinale, à savoir que la vox dei de la religion se mue ici - si ce jeu de mots latins est permis - en vox rei. Si, avec son Zarathoustra, Nietzsche n'ambitionnait rien moins que de créer une nouvelle religion, cette religion était bien évidemment une religion de l'immanence substituée à la transcendance, une religion de l'homme substituée à la religion de Dieu. Cette substitution doctrinale s'opère de manière d'autant plus efficace que Zarathoustra - en tant que discours - peut en l'occurrence se prévaloir du prestige immense du discours dominant de la culture occidentale depuis deux millénaires. Par ce coup de force discursif, toute la puissance performative de la prophétie religieuse est confisquée et réinvestie dans la parole la plus séculière et prosaïque qui soit: la radicalité de la fondation discursive se superpose à la radicalité de la révolution doctrinale. Et une nouvelle fois, cette dimension doctrinale, loin d'être simplement thématisée dans des concepts, se constitue comme signifiance, en marge et en amont des contenus, hors de l'œuvre elle-même, dans ses franges métadiscursives.

Notons que ce que Nietzsche écrit dans Ecce homo à propos de l'inspiration qui lui aurait dicté le Zarathoustra ne doit sans doute pas être pris au pied de la lettre. Cette inspiration fulgurante, contraignante, irrésistible, tout cela s'accorde fort mal avec ce que montrent les études menées sur les manuscrits: Nietzsche travaillait lentement, de façon on ne peut plus réfléchie, remettant cent fois l'ouvrage sur le métier. Les propos de Nietzsche dans Ecce homo n'ont probablement qu'une valeur documentaire très relative. Alors pourquoi de telles affirmations? Peut-être justement parce que Nietzsche - en même temps qu'une image de lui - construisait une signifiance discursive. Cette « fiction » sur l'œuvre, élaborée en marge de l'œuvre, participerait donc encore de l'œuvre.

Par ailleurs, toujours en ce qui concerne la constitution doctrinale, Ecce homo a plusieurs autres répercussions sur le Zarathoustra. L'une d'entre elles redouble et confirme ce que nous avons qualifié de "présupposé discursif ", présupposé construit par les mentions réitérées de "Zarathoustra » dans le métadiscours environnant. Ecce homo, œuvre rétrospective qui reconvoque et revisite plusieurs écrits antérieurs (dont Zarathoustra), suggère en effet, d'une autre façon, que la philosophie «déborde » l'œuvre l'individuelle. Plus de philosophie "mise en conserve ", mais un procès continu de création, une philosophie qui voudrait faire éclater la gangue des titres, des œuvres et des genres, qui voudrait en somme sortir d'elle-même pour se confondre en dernière instance avec la vie dans son ensemble.

Autre exemple: dans Ecce homo, Nietzsche évoque son combat intérieur, combat opposant I) l'inspiration irrésistible qui l'aurait traversé lors de la rédaction du Zarathoustra, et 2) les "forces défensives " (DefensivKräfte, KGW VI.3.340) qui auraient tenté de contenir ce déferlement à l'intérieur de lui-même. Or, très schématiquement, n'a-t-on pas ici comme une 
sorte de «miniature» du combat universel qui oppose la vie au nihilisme, les forces actives aux forces réactives ${ }^{20}$ ? Si tout est vie ou négation de la vie, la philosophie elle-même doit aller dans le sens de la vie ou en sens inverse. Aller en sens inverse de la vie revient à réduire le mouvement à l'immobilité, à enfermer la vie dans des notions immuables, ce que fait la philosophie "classique» qui participe en cela du nihilisme ambiant. Dans Ecce homo, Nietzsche a donc probablement voulu suggérer que sa philosophie (celle qu'il entendait transmettre dans son Zarathoustra) était, au sens le plus concret, coextensive à la vie, et non simplement théorie portant sur elle. Loin de se contenter d'évoquer in abstracto le combat vital de l' "actif» et du "réactif », cette philosophie veut montrer qu'elle émerge aussi elle-même de ce combat, un combat qui, en l'occurrence, se serait déroulé dans la tête du créateur-démiurge. L'activité même du philosophe, l'acte de créer et l'acte d'écrire hic et nunc sont, au même titre que tout le reste, performance vitale.

Mais, dira-t-on, ce que dit Nietzsche sur ce fameux combat intérieur n'a sans doute encore une fois rien à voir avec ce qui s'est passé réellement. Raison de plus, pensons-nous, pour postuler que les commentaires rétrospectifs de Ecce homo avaient un autre objectif: à partir des marges métadiscursives du Zarathoustra et de façon sans doute plus délibérée qu'il n'est communément admis, Nietzsche construisait une signifiance philosophique. Le jeu intertextuel et la dynamique discursive mis en place à partir de Ecce homo participent pleinement de la constitution doctrinale. Sans doute le vitalisme nietzschéen devait-il — ne fût-ce que pour rester cohérent avec luimême — s'inscrire dans un discours lui-même "vital ", un discours "débordant » au sens le plus littéral du terme.

\section{Le «discours-récit»}

Dans un deuxième temps, toujours dans le cadre de cette première étude portant sur le renouveau du discours philosophique, nous nous pencherons sur ce que nous qualifions de discours-récit. La philosophie, même si elle oblitère sa propre discursivité comme un facteur contingent, demeure un genre foncièrement discursif. Dans ses Problèmes de linguistique générale,

20. On pourrait d'ailleurs ajouter ici que ce phénomène, relevant de l'encodage et de la production de l'œuvre, semble avoir son équivalent du côté du décodage et de la réception de celle-ci. Comme le soutient Babette E. Babich (sans en tirer à notre sens toutes les conséquences philosophiques), Nietzsche aurait en effet cherché — au delà de la production - à programmer également la réception de ses propres écrits, qu'il aurait délibérément rendus peu accessibles, afin que s'opère un tri parmi ses lecteurs, sélectionnant justement le lecteur capable d'une lecture ardue et exigeante, assimilable à un combat: "Nietzsche's demanding prescrition for a reader is justified as a demand equally met on the author's side " " On Nietzsche's concinnity", in Nietzsche-Studien, internationales Jahrbuch für die Nietzsche-Forschung, op. cit., Bd I9, p. 74). Cela semble confirmer l'hypothèse que la philosophie de Nietzsche est indissociable d'une dynamique discursive (en l'occurrence métadiscursive) qui déborde de tous côtés l'œuvre individuelle et ses contenus thématisés. 
E. Benveniste parle de l' «objectivité » du récit qu'il oppose à la «subjectivité » du discours. Contrairement au discours, le récit est, selon Benveniste, détaché du sujet qui n'a pas à le prendre en charge: «Les événements semblent se raconter eux-mêmes ${ }^{21}$.» Le discours doit en revanche être assumé par un sujet du fait qu'il est lié au hic et nunc de l'énonciation.

Or on imagine mal une philosophie sans sujet, une philosophie sans un philosophe qui l'énonce et l'assume. Même si le sujet philosophique paraît effacé dans Zarathoustra, cet effacement n'est qu'un simulacre; il suffit pour s'en convaincre de voir à quel point ce sujet est omniprésent dans le métadiscours environnant. La philosophie est un genre foncièrement discursif; elle l'est depuis qu'elle s'est constituée comme telle aux origines de la pensée occidentale (notamment avec Platon), par démarcation face au mythe et au récit mythique. Pourquoi donc au final parler d'un discours-récit? Certainement pas pour réaffirmer à la suite d'innombrables interprètes de Nietzsche que le Zarathoustra appartiendrait à un genre hybride entre littérature et philosophie. Cette dénomination entend simplement suggérer que le discours nietzschéen intègre le récit, mais il l'intègre comme un moment philosophique. Loin d'être un simple indice de littérarité, ce récit «surdéterminé » devient aussi support de signifiances philosophiques. Nous nous proposons de recenser quelques modalités du phénomène.

\subsection{La «stratégie du retrait énonciatif»}

Nous qualifions le premier phénomène observé dans ce cadre de stratégie $d u$ retrait énonciatif. Dans le Zarathoustra, on voit effectivement s'effacer l'une après l'autre les instances qui animent habituellement le récit: auteur, narrateur et même, si paradoxal que cela puisse paraître, le personnage. L'auteur du Zarathoustra, l'énonciateur philosophique, alias Nietzsche, est d'emblée un énonciateur effacé: dès le début du prologue "Als Zarathustra dreissig Jahre alt war» (KGW VI.I.5), et avant même d'avoir fait la moindre «apparition ", cet énonciateur délègue la parole à un narrateur ${ }^{22}$. Or ce narrateur lui-même est - selon l'expression d'Alain Rabatel — un "narrateur effacé ${ }^{23}$ qui n'apparaît qu'en pointillés dans la diégèse. Il est vrai que ce narrateur parle à la fin de chaque discours de Zarathoustra, prononçant la formule-leitmotiv qui est aussi le titre de l'œuvre: "also sprach Zarathustra». Mais si l'on y regarde de plus près, le narrateur, quand il parle, ne parle en fait que pour signaler qu'un autre a parlé (verbes locutoires), c'est-à-dire pour manifester son effacement derrière les paroles qu'il rapporte. Cette

21. Problèmes de linguistique générale, Paris, Gallimard, II, I974, p. 262.

22. Cette attitude de distanciation de l'énonciateur philosophique (l'auteur) a été soulignée notamment par Werner Stegmaier: "Nietzsche entzieht der Erzählung von den Lehren Zarathustras einen erkennbaren Autor, der die Verantwortung für sie übernimmt» ("Anti-Lehren, Szene und Lehre » in Volkert Gerhardt (Hg.), Also sprach Zarathoustra, op. cit., p. 199).

23. Une histoire $d u$ point de vue, Recherches textuelles $n^{\circ} 2$, Publication du Centre d'études linguistiques des textes et des discours, Université de Metz, I997, p. I63. 
présence du narrateur dans la formule-leitmotiv n'est donc finalement qu'une présencelabsence, rendue encore plus improbable par son côté incantatoire. Quant au personnage, Zarathoustra, il parle continuellement, au point que ses discours «saturent» l'œuvre. Zarathoustra déclare d'ailleurs lui-même: "Mund bin ich worden ganz und gar " (Das Kind mit dem Spiegel, KGW VI.I.IO2). Pourtant, comme cette dernière formule le suggère, il est indéniable que les paroles de Zarathoustra échappent à son contrôle et le submergent passablement. Il s'épuise dans les paroles qu'il profère en permanence, sans que ces paroles émanent pour autant vraiment de lui. On pourrait dire à propos du Zarathoustra: "ça parle», mais on ne peut au final éluder la question: "Qui parle vraiment ? Z Zarathoustra est une figure diaphane, nul ne connaît son aspect, il parle toujours, mais il ne parle jamais de lui, il se dilue en quelque sorte dans l' "altérité" des sujets qu'il traite par myriades. Dans la terminologie de Maingueneau, nous dirions que Zarathoustra possède un "ethos vide ${ }^{24}$ ». Le personnage est sans consistance; même son envergure intellectuelle supposée est finalement peu crédible. Son aptitude à faire surgir en un tournemain l'univers entier face à des auditoires subjugués laisse perplexe. On ne peut se défaire de l'impression que Zarathoustra est habité par une puissance qui le dépasse et parle à travers lui. C'est ce que semblent d'ailleurs confirmer ses phases extatiques, ses absences cataleptiques, tout comme le fait qu'il soit à plusieurs reprises dépossédé de ses propres paroles, par exemple dans le chapitre "Der Genesende", où les animaux de Zarathoustra doivent formuler à sa place la pensée de l'éternel retour (KGW VI.I.268269). Bref, après l'effacement de la fonction-auteur et l'effacement de la fonction-narrateur, il faut prendre acte de l'effacement de la fonctionpersonnage.

\subsection{Le retrait énonciatif comme philosophème}

Or, en ce qui concerne les signifiances, ce retrait énonciatif possède indéniablement un effet fondateur. Détaché de la chaîne énonciative, le message de Zarathoustra s'affranchit de toute contingence communicationnelle, devenant auto-référentiel et auto-performatif. La parole de Zarathoustra ne vient de nulle part et elle fait jaillir du néant un univers inédit, sans rapport aucun avec quoi que ce soit d'existant: "Neue Wege gehe ich, eine neue Rede kommt mir » (Das Kind mit dem Spiegel, KGW VI.I.IO2). Zarathoustra le dit lui-même: la parole qu'il profère «lui vient» d'ailleurs, propos qui rappelle évidemment ce qu'écrit Nietzsche dans Ecce homo à propos de sa propre inspiration venue comme un «don du ciel» (cf. supra); du métadiscours au discours-récit, les signifiances convergent. On pourrait certes objecter à cela qu'en perdant ses références énonciatives (énonciateur philosophique/

24. «L'énonciation discursive comme institution philosophique ", in Langages $n^{\circ} 119$, L'analyse du discours philosophique, op. cit., p. 57. 
narrateur/personnage), le message perd du même coup ses ancrages réels, qu'il se «fictionnalise». Pour Benveniste, un tel message serait à l'opposé de ce qu'il nomme "discours "; il correspondrait en revanche parfaitement à sa définition minimaliste du récit, où "les événements semblent se raconter eux-mêmes ». Est-ce à dire que le Zarathoustra n'est plus « discours philosophique ", mais simple "fiction littéraire" ? Une telle hypothèse serait intenable; suffisamment d'indices convergents l'attestent: la "fictionnalité » du Zarathoustra, tout comme le retrait énonciatif qui la génère, est une signifiance philosophiquement orchestrée. Le philosophe est toujours là et bien là, même s'il feint de s'effacer, mais, pour des raisons que nous avons déjà évoquées et sur lesquelles nous reviendrons, il veut gérer la production du sens de façon non conventionnelle: aux signifiés conceptuels classiques, il entend substituer des effets langagiers et discursifs. Le Zarathoustra de Nietzsche se déploie donc sur deux strates distinctes: pour être récit fictionnel, il n'en est pas moins discours; un discours d'un type nouveau qui ne se positionne pas «massivement» — comme c'est le cas du discours philosophique traditionnel - en contrepoint de la fiction, mais intègre au contraire celle-ci à sa démarche comme philosophème. C'est en ce sens-là que nous avons parlé de discours-récit.

\section{3 «Défilé de masques » et «métaphore énonciative»}

Mais ces instances énonciatives, qui s'effacent les unes derrière les autres et s'effacent derrière le message, évoquent aussi ce que nous qualifions de défilé de masques. De fait, pour Nietzsche tout est masque: "Jeder tiefere Geist braucht eine Maske» (KGW VI.2.54), ou encore: "Jede Meinung ist ein [...] Versteck, jedes Wort eine Maske» (KGW VI.2.244), écrit-il dans Par-delà le Bien et le Mal. La vérité n'est jamais rien d'autre qu'une illusion non encore dévoilée, et ce qui se lit en filigrane du défilé de masques, c'est bien sûr très schématiquement - ce qu'il est convenu d'appeler le perspectivisme. Ce perspectivisme, non «théorisé », se "montre» en l'occurrence dans le jeu du retrait énonciatif, lequel participe donc - en termes de signifiances - à la constitution doctrinale.

Par analogie avec la métaphore qui, chez Nietzsche, est un défilé d'images (les métaphores s'appellent sans fin les unes les autres dans ce que nous avons qualifié dans une autre étude de «jeu métaphorique» ou encore de «métaphore processuelle ${ }^{25}$ ), on peut parler ici de «métaphore énonciative». Comme les images, les instances énonciatives s'effacent les unes derrière les autres et se succèdent, suggérant le perspectivisme, le primat de l'illusion sur la vérité, du mouvement sur l'immobilité, du devenir sur l'être. En revêtant «personnellement » ces masques successifs au lieu de se retrancher derrière le masque figé du sujet philosophique souverain, Nietzsche

25. Lectures d'une œuvre, Also sprach Zarathoustra, ouvrage collectif coordonné par G. Merlio, Paris, Éditions du Temps, 2000, p. 210 sq. 
suggère en somme qu'il vit lui-même ce perspectivisme, non dans un "ailleurs » théorique, mais dans l'exercice hic et nunc de sa philosophie.

\subsection{Retrait énonciatif et «corps logocisé»}

Mais la métaphore énonciative joue aussi d'une autre façon. Il y a en effet chez Zarathoustra un paradoxe: comment expliquer que le philosophe, qui se proclame apologiste du corps, puisse proférer une parole à ce point désincarnée? Car tel est bien l'effet produit par le retrait énonciatif: plus d'auteur, plus de narrateur, plus de personnage, seul reste un message sui-référentiel qui «traverse" en quelque sorte ces instances devenues diaphanes. Pour expliquer cette apparente contradiction, il faut bien saisir qu'entre « corps » et « parole », il n'y a pas chez Nietzsche de solution de continuité. Pour le philosophe, le corps n'est jamais corps chosifié, mais ce que l'on pourrait qualifier de «corps ouvert». Pour rester corps au sens nietzschéen, le corps doit constamment "devenir », se transporter vers un autre que lui-même. Il doit, au sens le plus littéral du terme, se "métaphoriser», métaphorisation qui est précisément rendue possible par la parole, seule apte à l'affranchir de sa choséité. Cet indéfectible lien entre le "physique» et le «langagier» est d'ailleurs évoqué dans l'essai De la vérité et du mensonge au sens extra-moral, où Nietzsche retrace la genèse de la première métaphore: une excitation nerveuse se transforme en image donnant la première métaphore, laquelle est à son tour transformée en son, engendrant la deuxième métaphore ${ }^{26}$. C'est dans ce contexte que nous parlons de corps logocisé. En somme, la vie est foncièrement parole, parole métaphorique - et ne fût-ce que pour ne pas rester figée sans suite ni écho dans le silence et le néant philosophique d'un corps chosifié27. Le corps, s'il est tout pour Nietzsche, est un corps " ouvert », transitif, c'est-à-dire un corps non pas logicisé puisqu'il n'y a ni logique, ni limite, ni finalité à ce transit permanent du corps - mais un corps métaphorisé et logocisé. La métaphore énonciative sert donc de support à cette autre signifiance philosophique.

\subsection{La parole «corporéisée»}

Mais si le corps - pour rester corps - doit, nous dit Nietzsche, devenir parole, s'interpréter, ce corps se perdrait aussi s'il devenait exclusivement

26. «Ein Nervenreiz, zuerst übertragen in ein Bild! Erste Metapher; Das Bild wird nachgeformt in ein Laut! Zweite Metapher " (Über Wahrheit und Lüge im aussermoralischen Sinne, KGW III.2.373).

27. On a souvent noté le rapport étroit qui existe entre volonté de puissance et interprétation. Wolfgang Müller-Lauter, soulignant l'étendue de la notion d'interprétation chez Nietzsche, postule une pluralité de "volontés de puissance" auxquelles correspondraient autant d' «interprétations": "Alle Willen zur Macht legen aus, interpretieren" ("Nietzsches Lehre vom Willen zur Macht», Nietzsche-Studien, Internationales Jahrbuch für die Nietzsche-Forschung, op. cit. Bd 3, p. 43). Le même auteur écrit dans un esprit similaire: «Noch jedes 'Geschehen' besitzt 'interpretativen' Charakter» (ibid.). Il appartient au corps de s'interpréter, de "fuir» sa corporéité dans l'interprétation et son succédané, la métaphore, tous deux des succédanés du logos. 
parole. La métaphorisation n'est pas l'abstraction, et le référent corporel ne doit en aucun cas se diluer. Autrement dit, le mouvement qui mène de l'«excitation nerveuse » (Nervenreiz) à l'«image» (Bild) et au "son» (Laut) doit à tout moment pouvoir s'inverser. Le physique et le langagier doivent rester dans un rapport réversible; aux yeux de Nietzsche, le corps n'est ni chose ni parole, mais va-et-vient constant de l'un à l'autre. Si - comme nous venons de l'exposer - la métaphore énonciative retire les instances, il n'en est pas moins vrai qu'elle les rétablit aussi dans leurs rôles. De fait, le message sui-référentiel ("ça parle») ne domine sans partage que dans les parties II et III de l'œuvre, largement occupées par les seuls discours de Zarathoustra juxtaposés les uns aux autres. Dans la quatrième partie, on note en revanche un retour perceptible du narrateur. Le récit, précédemment on ne peut plus «mimétique » (discours de Zarathoustra rapportés tels quels à l'état brut), fait une part plus large à la diégèse.

De même, Zarathoustra, figure jusque-là diaphane traversée par un flux de paroles, semble devenir moins inconsistant. Pour la première fois dans l'œuvre, au lieu de s'abandonner au discours déferlant qui l'emporte, Zarathoustra se met à réfléchir à ce discours, qu'il utilise même de manière tactique: "Dass ich von Opfern sprach und Honig-Opfern, eine List war's nur meiner Rede» (Das Honig-Opfer, KGW VI.I.292). On remarque aussi, dans le même discours, que Zarathoustra a désormais le temps de parler: "wir haben zum Reden schon Geduld und Zeit und Überzeit» (KGW VI.I.294). Lui, qui antérieurement n'avait le temps de parler qu' «au présent » sans la moindre distance, son temps de parole se confondant pratiquement avec son temps de vie, semble ici prendre un certain recul face à cette urgence du «parler ${ }^{28}$. Il n'est plus possédé par le temps de la parole; c'est lui qui désormais possède largement ce temps (Überzeit).

En bref, dans cette quatrième partie, la parole devient moins évanescente; les instances énonciatrices (tout au moins le narrateur et le personnage) reprennent littéralement corps. Le mouvement de logocisation du corps, amorcé avec le retrait des instances énonciatives, est, sinon inversé, du moins relativisé. La référence au corps s'estompe, mais ne s'efface pas. À travers la métaphore énonciative, c'est en fait un double mouvement inverse qui se dessine: mouvement du corps vers la parole (corps logocisé), mais aussi retour de la parole vers le corps (parole corporéisée). Le traitement de l'énonciation rejoint donc ici encore une composante doctrinale.

Dans ce contexte, il est important de souligner que la métaphore énonciative - en tant que signifiance philosophique - ne doit pas être inter-

28. Ce qui va de pair, soit dit en passant, avec le fait que dans cette quatrième partie Zarathoustra ne recherche plus activement ses auditoires, mais entend les laisser venir à lui: " he still thinks of himself as a prozelitizer, though he now expects that men may come up to him» (Nehamas, Alexander, "For whom the Sun shines", in Volkert Gerhardt (Hg.), Also sprach Zarathustra, op. cit., p. I83). 
prétée comme un indice de "déconstructivisme». Il n'est pas question de dire, à la suite d'une certaine école d'exégèse nietzschéenne en vogue dans les années soixante-dix, sous l'influence entre autres des travaux de Derrida, que Nietzsche aurait «libéré » la parole parce qu'il aurait renoncé à communiquer le sens, son discours devenant une sorte de jeu esthétique parfaitement gratuit. Encore une fois, le discours nietzschéen ne se dilue pas dans une vague littérarité. L'intention philosophique n'est jamais perdue de vue. La logocisation du corps relève sans doute d'une forme de déconstruction, mais cette déconstruction a pour pendant une reconstruction articulée autour du corps physique. C'est ce qu'un auteur comme Lothar Jordan désigne sous le nom de «Rückbezüglichkeit ${ }^{29}$. Pour Nietzsche, le corps est ce pôle d'attraction - que la philosophie doit tenir à distance pour ne pas se figer dans un concret illusoire - mais auquel elle doit toujours revenir pour ne pas s'égarer dans l'abstrait ou l'insignifiant. Il y a chez Nietzsche cette idée fondamentale: un organisme "interprète» son environnement en fonction de ses intérêts vitaux, et cette interprétation «se souvient » des interprétations antérieures. Bref, à l'arrière-plan des idées, à l'arrière-plan des mots, il y a la mémoire du corps. Ici, la logocisation du corps touche donc à cette limite qu'est la corporéisation du logos. La déconstruction, si elle a bien lieu, est elle-même le produit d'un discours en construction autour d'un objet en construction qui est la vie organique.

\section{Le Zarathoustra de Nietzsche comme renouveau du discours philosophique: bilan}

En faisant le total des signifiances liées au métadiscours et au discours-récit, nous en sommes arrivé à la conclusion que Nietzsche initiait véritablement un renouveau du discours philosophique. Premier constat: la dynamique discursive laissée hors champ par la philosophie «classique» est rétablie dans ses droits. Très schématiquement, si l'on remonte aux origines platoniciennes (ou préplatoniciennes), tout semble se passer comme si le dualisme bien connu, consacrant la séparation de l'essence et de ses manifestations, structurait aussi dès le départ le mode d'exposition de la philosophie: avec Platon, c'est la philosophie tout entière qui bascule du côté du logos pour devenir exclusivement quête du "vrai». Or, pour Platon, le «vrai» ne peut se donner que dans l'idée pure. Tout le reste est relégué dans le domaine du «faux» qui est par définition étranger à la philosophie, cela incluant toutes les manifestations contingentes de l'idée, et en particulier ses modes d'actualisation dans la langue et dans le discours ${ }^{30}$. C'est en ce sens que nous enten-

29. "Nietzsche Dekonstruktionist oder Konstruktivist », in Nietzsche-Studien, Internationales Jahrbuch für die Nietzsche-Forschung, op. cit., Bd 23, p. 232.

30. L'idée par définition ne se «raconte» pas, puisque ce qui se «raconte», notamment sous forme de récit, relève de la fiction liée au muthos, soit précisément ce à quoi la philosophie, en tant que discours, s'oppose. On pourrait bien entendu objecter à cela que les œuvres de 
dons la formule "épiphanie de la vérité »: en termes de tendances, l'histoire de la philosophie ressemble incontestablement à un cortège d'idées, seules les idées importent, l'enveloppe discursive et langagière, elle, importe relativement peu $^{31}$. Discours de la vérité, la philosophie ne se pose pas radicalement la question de la vérité du discours, puisque cette question la mettrait en porte-à-faux, puisqu'elle la jetterait en quelque sorte en dehors d'ellemême, hors des limites du genre posées dès le départ (notamment avec Platon). Avec le Zarathoustra de Nietzsche, cette tendance s'inverse donc pour la première fois du tout an tout.

De là, sans doute, l'importance des signifiances fondatrices qui forment un véritable dispositif visant à légitimer et positionner le Zarathoustra dans l'interdiscours. De là également, et c'est sans doute la chose la plus importante, un faisceau de signifiances convergentes participant à la constitution de la doctrine. Nietzsche énonce en substance: la vie est un continuum irréductible, la volonté de puissance ne se divise pas, le devenir n'est pas l'être. Les stratégies discursives mises en œuvre (l'exploitation des marges métadiscursives, le discours-récit) font indéniablement écho à ce

Platon sont elles-mêmes des récits dialogués. Mais ces dialogues, loin d'être l'annonce inaugurale d'une prise en compte d'une discursivité dialogale comme philosophème, sont probablement aussi le dernier mythe annonçant précisément la fin du mythe et la naissance (ou renaissance) de la philosophie. Par ailleurs, les dialogues platoniciens sont placés sous le signe de la dialectique socratique, dialectique qui, pour Platon, est elle-même finalisée par le principe suprême qu'est l'idée du Bien. Comme l'écrit E. Chambry en préalable à sa traduction du Théétète et du Parménide: "Cette méthode socratique, Platon l'étend au domaine des Idées, pour les atteindre elles-mêmes et monter des Idées inférieures à l'Idée du Bien [...] Chaque hypothèse est un degré qui nous hausse vers l'Idée (Platon, Théétète, Parménide, traduction, notices et notes par Emile Chambry, Paris, Flammarion, I967, p. I6). «Le fond [des dialogues de Platon] est toujours une question philosophique, et le but, la recherche d'une vérité au moyen de la dialectique» (ibid., p. 23). En somme, les dialogues ne sont chez Platon que des constructions inféodées à la quête de la vérité, ils ont plus l'allure de syllogismes «illustrés » que de véritables récits. Dans la plupart des dialogues, l'histoire se résume à une confrontation de points de vue, soutenus par différents personnages, et au final seul le point de vue manifestant le «vrai » subsiste, généralement soutenu par Socrate. Les dialogues platoniciens ne sont donc souvent qu'une mise en scène du "vrai » et du "faux », dont le dénouement est toujours la consécration du «vrai » (et l'exclusion du «faux»). Le «vrai» doit être exposé, telle est la chose essentielle; la discursivité dialogique n'est finalement qu'accessoire, peut-être un reliquat de la phase où mythe et philosophie se confondent encore. C'est là une possible explication à l'attachement insistant de Platon à cette forme dialogique, attachement qui a parfois surpris ses exégètes: «Platon n'a pas conçu d'autre méthode que la dialectique socratique, et il l'a gardée toute sa vie, même lorsque, semble-t-il, une exposition suivie [...] eût donné à ses démonstrations plus de force et de netteté » (ibid., p. 23). En tout état de cause, dès Platon, la voie semble — tendenciellement - ouverte à ce type d' "exposition suivie " qui évacuera de plus en plus le récit (et, paradoxalement, le dialogue) comme phénomènes parasites.

31. Nous insistons sur le fait qu'il ne s'agit pas d'une règle absolue, nous ne prétendons pas que l'écriture philosophique fût totalement dénuée d'incidence avant Nietzsche. Simplement: avec Nietzsche, le phénomène s'accentue au point d'induire un changement de paradigme. 
fonds doctrinal. Nietzsche façonne Zarathoustra non comme une œuvre, mais un processus en gestation. Du Gai Savoir à Ecce homo an passant par les Dithyrambes à Dionysos, cette œuvre n'en finit pas de s'écrire de multiples manières, comme si elle voulait sortir de tous les cadres réducteurs pour se confondre avec ce qu'elle clame et proclame: la vie. C'est aussi pour mieux correspondre aux exigences de la vie que Nietzsche se tourne vers les origines "préphilosophiques ", ce creuset où tout se mêle, dont la philosophie s'est démarquée au départ, là où le vrai et le faux, la réalité et la fiction cohabitent, dans l'indivis, là où le devenir n'est pas encore l'être, là où l'image vivante ne s'est pas encore figée en concept, là où le muthos (homérique ou hésiodique) n'est pas encore devenu logos. Il faut totaliser, et non réduire les perspectives, tel est peut-être le fin mot du perspectivisme de Nietzsche. En ce sens, le récit convoqué en philosophie par Nietzsche n'interfère pas avec le discours (réducteur); il le «supplémente " philosophiquement.

Dans le même esprit, Nietzsche ne s'exclut pas de sa propre philosophie, il refuse de s'inscrire dans le mouvement «épiphanique» ambiant, appliquant en quelque sorte le perspectivisme in vivo à sa propre activité, qui consiste à philosopher. Ainsi, se mettant en quelque sorte lui-même en abyme, il arbore avec conséquence les masques changeants qui recouvrent et recouvriront à l'infini la réalité des choses. Bref, Nietzsche ne pouvait pas écrire sa philosophie en l'enfermant dans une œuvre unique, sans la faire déborder sur toutes les autres œuvres et au delà des œuvres; Nietzsche ne pouvait pas écrire sa philosophie sans réfléchir simultanément sa propre action consistant à écrire, sans réfléchir et anticiper la réception de ses textes, sans rendre «vitale» sa propre écriture, sans faire signe vers les sources, vers la matrice mythique où tout s'est engendré, y compris le discours réducteur sur le «Vrai». Ce double geste spéculaire et généalogique est indissociable de sa philosophie. Il s'affirme pleinement avec le Zarathoustra.

La question de la spécularité philosophique, soulevée par nos propres thèses, renvoie par ailleurs à la problématique souvent discutée de «la compréhension du perspectivisme» qui, comme l'écrit Jean Granier, «nous arrache au perspectivisme $»^{32}$. Toute l'aporie réside dans le fait qu'on ne peut affirmer le perspectivisme qu'en adoptant soi-même une certaine perspective. En dernière extrémité, tous nos efforts de décentrage par rapport à une quelconque perspective donnée s'échouent dans le langage que nous sommes condamnés à utiliser pour formuler nos propositions, le langage nous imposant en quelque sorte en dernier ressort sa perspective. J. Granier formule donc son hypothèse finale, à notre avis extrêmement féconde, d'un métalangage. Mais pour Granier le métalangage dont Nietzsche ferait usage serait un instrument non maîtrisé; selon lui, Nietzsche renoncerait au final à «la possibilité même d'une interprétation de l'interprétation", ruinant «le

32. Granier, Jean, Le problème de la vérité dans la philosophie de Nietzsche, Paris, Seuil, I966, p. 606. 
fondement de son propre discours ${ }^{33}$. À nos yeux le métalangage dont parle Granier ne saurait se limiter, comme il l'énonce, à la recherche d'un fondement; ce métalangage chercherait plutôt à suggérer sa propre relativité et son propre inachèvement. Car le métalangage dont il est ici question est dynamique: il y a en quelque sorte toujours un "méta " au delà du "méta ". Or, dès lors que l'on prononce un tel mot: «métalangage» ou "perspectivisme ", on pervertit le phénomène, fatalement, on immobilise ce qui est foncièrement mouvant. C'est donc encore dans le non explicite, dans des signifiances discursives révélant leur propre relativité, que le perspectivisme se manifeste. Si l'idée d'un métalangage est féconde, pour nous, ce métalangage n'a rien d'un échec; c'est au contraire une construction sciemment orchestrée. Nietzsche ne cherche pas à «thématiser» (vainement) l' "espace du discours ${ }^{34}$, comme le suggère Granier; il exploite directement cet espace discursif (et sa dynamique) sous forme de signifiances.

Mais ces conclusions doivent aussi être mises en regard d'un autre interprète incontournable de Nietzsche avec lequel nous sommes tout à fait d'accord sur de nombreux points: Eric Blondel. Blondel voit lui aussi chez Nietzsche un renouveau de l'écriture philosophique, mais la dynamique qu'il prête à l'écriture nietzschéenne se situe à ses yeux dans l'«écart stylistique». Blondel qualifie, par exemple, le texte de Nietzsche de «discontinu et aphoristique", opposé "au projet architectonique du discours philosophique classique ${ }^{35}$. Il parle encore d'une prolifération de tropes, de figures, allusions, mythologismes, paronomases, etc. ${ }^{36}$, qui démarquent le style du texte nietzschéen. Il évoque aussi l'absence d'unité de ton: burlesque, sérieux, ironie, tragique, grandiloquence, théâtralisme, et d'unité de genre: proverbes, maximes, paraboles, démonstrations philosophiques, dialogues dramatiques, psaume, prière ${ }^{37}$, etc. Bref, Blondel insiste sur l'écart stylistique et générique, et ses constats sont tous très pertinents et novateurs sur ce point. Mais pour l'auteur, le renouveau apporté par Nietzsche a trait au texte, le «discours» restant pour lui synonyme de fixité et d'attachement aux normes du genre. À nos yeux, le discours (ce que nous appelons aussi la "discursivité») est plutôt procès en marche, le texte n'étant que le point d'aboutissement et la cristallisation de ce procès. Si, comme le pense J. M. Adam, le discours est le texte plus ses conditions de production, le texte étant à l'inverse le discours moins ses conditions de production, la dynamique est bien du côté du discours. De la même façon qu'il a exploité l'écart stylistique par rapport aux normes du genre, Nietzsche a, à notre sens, aussi exploité l'écart discursif. Mais au-delà des divergences de vocabulaire, il

33. Ibid.

34. Ibid., p. 607.

35. Nietzsche, le corps et la culture, Paris, PUF, Philosophie d'aujourd'hui, I986, p. 22.

36. Ibid., p. 28.

37. Ibid., p. 28-29. 
nous semble que les conclusions d'Eric Blondel et les nôtres se complètent plutôt qu'elles ne s'opposent.

\section{Zarathoustra comme «performance» philosophique}

\section{Méthode et hypothèses}

Dans la foulée de ce dernier travail sur le Zarathoustra une autre idée nous est venue: si Nietzsche refond et ressuscite la dynamique du discours philosophique, pourquoi le Zarathoustra ne pourrait-il être, dans une certaine mesure, concevable comme un acte de langage (au sens d'Austin)? Un discours «vital» dans les modalités de sa production ne devait-il pas chercher à être «vital» dans son influence sur autrui. Pourquoi dès lors n'a-t-on jamais cherché à appréhender Zarathoustra sous l'angle de la fonction illocutoire perlocutoire?

Il est indéniable que le héros Zarathoustra, s'il agit fort peu lui-même, veut faire agir et réagir les autres. On pouvait donc conjecturer sans grand risque que la philosophie de Nietzsche, qu'il est convenu de définir comme une philosophie du vouloir, devait aussi chercher à «faire vouloir». La belle formule qui clôture l'épilogue du Gai Savoir ne suggère-t-elle pas d'ailleurs cette piste: "Aber was ibr zu hören bekommt, ist wenigstens neu; und wenn ihrs nicht versteht, wenn ihr den Sänger nicht versteht, was liegt daran! [...] Um so deutlicher könnt ihr seine Musik und Weise hören, um so besser auch nach seiner Pfeife - tanzen. Wollt ibr das?..." (KGW V.2.320). N'est-ce pas au Zarathoustra - cette œuvre si «liée» au Gai savoir (cf. supra) que ces phrases correspondent le mieux? Sous le couvert d'une métaphore musicale, elles disent que le lecteur est incité à suivre le philosophe, mais son adhésion n'est pas contrainte; cette adhésion ne peut être qu'un acte de vouloir propre. "Wollt ihr das?..." , l'apostrophe énigmatique qui clôture l'épilogue du Gai Savoir laisse perplexe. Le «vouloir», mis en exergue, n'est-il pas la seule suite possible à donner à ces mots? La parole cesse (points de suspension dans la citation), le vouloir commence. Zarathoustra peut lui-même commencer à parler! Bref, il nous semblait que l' "appel », plus ou au moins autant que l' «information» (ces termes étant entendus au sens de la pragmatique), devait tenir dans Zarathoustra une place essentielle et qu'un rapport existait entre appel, vouloir et faire vouloir.

Sur cette base, notre questionnement central sera donc: comment se manifeste concrètement l'appel dans le texte? Peut-on mettre en relation ces manifestations avec la philosophie de Nietzsche? Nous tentons d'y répondre dans ce second travail d'envergure sur Zarathoustra, nous appuyant en cela sur la notion de «fonction appellative» décrite par la pragmatique textuelle moderne. Implicitement nous faisons bien sûr à nouveau appel à la notion de signifiance: si le texte de Nietzsche est effectivement à comprendre comme un appel, cela n'est dit explicitement nulle part; une telle conclusion ne peut donc être que le résultat d'une interprétation. Bref, l'objectif concret 
était de rechercher dans le texte les indices de fonctions illocutoires, avec en ligne de mire les indices de «fonction appellative». Très schématiquement, nous opérons un distinguo entre les indices d'illocution directe et les indices d'illocution indirecte. Concernant l'illocution directe, nous nous appuyons notamment sur E. U. Große, qui énumère un certain nombre d'indices d'identification de l'appel, indices qu'il qualifie de voluntas-Signale («indices de volition»). Parmi eux, il y a par exemple les «formules introductrices» qu'il qualifie de "Präsätze» et qu'il regroupe en quatre catégories: I) formules introductrices exprimant le «vouloir ", le «souhait», l'«intention» et la «préférence» (wollen, wünschen, beabsichtigen, vorziehen); 2) formules introductrices exprimant la «requête ", l' «exigence», la «réclamation» (verlangen, fordern, beantragen); 3) formules introductrices exprimant l' "ordre", la « recommandation", la "proposition ", la "prière» et l' "avertissement" (befehlen, empfehlen, vorschlagen, bitten, warnen); 4) formules introductrices exprimant la «demande» (fragen $)^{38}$. Parmi les «indices de volition» (voluntas-Signale), il en est également qui ne sont pas des «Präsätze», mais des formes de phrases (modes verbaux: impératif, infinitif, interrogatif) ${ }^{39}$. Hormis les indices de volition, la seconde catégorie d'indices d'identification de l'appel, toujours en illocution directe, sont selon Grosse les indices de nécessité qu'il identifie comme: I) structures attributives (Präsätze): "es ist notwendig, unentbehrlich» (il est nécessaire, indispensable)/vs/ "unzulässig, verboten, etc.» (illicite, interdit, etc.); 2) des tournures infinitives du type "hat...zu» (avoir à...); 3) les verbes modaux «sollen» et "müssen». Sans pouvoir ici exposer le détail de notre méthode, nous avons donc, dans le texte de Nietzsche, recensé et analysé dans leurs nuances les indices de volition et de nécessité qui indexent l'appel de façon directe.

Par ailleurs, comme il est évident qu'on ne peut réduire la fonction appellative d'un texte à la simple somme arithmétique de tels indices, nous avons tenu compte (sans doute même de façon prioritaire) des indices appellatifs indirects qui se manifestent dans et par le texte de manière globale, diffuse ou médiate. Toujours selon Große, il s'agit: I) du "facteur appel» (Appellfaktor), 2) des "présignaux» (Präsignale), 3) des "règles de l'agir» (Handlungsregeln). Le «facteur appel» se manifeste: a) par l'"abondance de mots et de tournures à caractère évaluatif ${ }^{40}, b$ ) par «l'abondance des figures rhétoriques ${ }^{41}$. Les "présignaux » sont des éléments (souvent d'ordre paratextuel) qui servent à classer d'emblée le texte dans telle ou telle catégorie (titre, genre, etc. $)^{42}$. Quant au troisième critère de Große, les « règles de

38. Ernst Ulrich Große, Text und Kommunikation, Stuttgart, Berlin, Köln, Mainz, Verlag W. Kohlhammer, 1976, p. 88-89.

39. Ibid., p. 86.

40. Ibid., p. I 8.

41. Ibid.

42. Ibid., p. 20 sq. 
l'agir" (Handlungsregeln), il renvoie à ce qui est commun aux partenaires de la communication: "die Kommunikationspartner handeln nur [...] nach ibnen und verstehen sie nur dann, wenn ibnen diese Regeln gemeinsam sind", ce qui suppose une "reconnaissance» mutuelle (Anerkennung), ainsi que des "conventions» (Konventionen) ${ }^{43}$. Au final Große propose une équation regroupant l'ensemble de ces éléments: "Textfunktion $=( \pm$ Handlungsregeln $)$ ( \pm Präsignal) ( \pm Appellfaktor) + metapropositionale Basis + Propositionstyp ${ }^{44}{ }^{\prime}$.

C'est - avec de multiples adaptations, nuances et compléments - ce modèle que nous avons appliqué au texte de Nietzsche. Sous le nom de «structures illocutoires complexes", nous insistons notamment sur ce que K. Brinker qualifie de "formes fondamentales de développement thématique (Grundformen thematischer Entfaltung), distinguant le «développement thématique descriptif» (deskriptive Themenentfaltung), le "développement thématique explicatif» (explikative Themenentfaltung) et le "développement thématique argumentatif " (argumentative Themenentfaltung) ${ }^{45}$, toutes potentiellement associées à l'appel (rubrique "Appelfaktor» de Große). Nous y ajoutons une autre forme de développement thématique: le récit, qui est dans beaucoup de cas un modèle de déploiement thématique figurativisé ${ }^{46}$ et peut donc être associé sous divers aspects à l'appel, même si cet appel se démarque assez nettement de celui qui est associé au modèle argumentatif. Ainsi, selon J. F. Jeandillou, «l'ordre du récit se trouve [...] soumis à une constante tension pragmatique qui articule les événements nouveaux avec leurs implications potentielles [...] les instructions données au lecteur l'amènent à interpréter les faits en révisant sans cesse son jugement ${ }^{47}$ ». Il y a incontestablement dans ce suspens propre à tout récit qui maintient l'interlocuteur «en haleine» une dimension illocutoire apparentée à l'appel. Parmi ces structures illocutoires complexes qui engagent le texte entier (on parlera aussi à leur propos d'indices macrocommunicationnels), nous avons retenu tout particulièrement un schéma actantiel (relations sujet/objet//destinateur/ destinataire), qualifié par Nicole Everaert-Desmedt de «contrat de séduction ${ }^{48}$. Dans ce type de schéma, le récit génère ce que R. Girard qualifie de "désir mimétique » ${ }^{49}$, le désir d'un sujet pour un objet transmis par un

43. Ibid., p. 22. Ces «règles de l'agir» semblent proches des «maximes conversationnelles » rendues célèbres par Grice, la «reconnaissance» en particulier, faisant pendant au principe de "coopération", qui est selon Grice la base élémentaire de toutes les autres maximes.

44. Ibid., p. I 16.

45. Klaus Brinker, Linguistische Textanalyse, eine Einführung in Grundbegriffe und Methoden, Berlin, Erich Schmidt Verlag, Grundlagen der Germanistik, 4. Auflage, I997, p. 63 sq.

46. Greimas et Courtés appellent "figurativisation" la "conversion des thèmes en figures" (Algirdas Julien Greimas et Joseph Courtés, Dictionnaire raisonné de la théorie $d u$ langage, Paris, Hachette Université, I979, I, p. I78).

47. Jean-François Jeandillou, L'analyse textuelle, Paris, Armand Colin, 2007 (I997), p. 148 .

48. Sémiotique du récit, Bruxelles, De Boeck Université, Prismes Méthodes 8, I988, p. 50. 49. René Girard, La violence et le sacré, Paris, Grasset, I987, p. 204. 
destinateur à un destinataire à l'intérieur du récit pouvant, par exemple, susciter le désir d'un autre sujet pour un autre objet intradiégétique, ou encore - par " trope communicationnel ${ }^{50} »$ - correspondre au désir d'une instance extradiégétique (l'auteur), transmis à un destinataire lui-même extérieur au récit (le lecteur). Nous y reviendrons.

Pour résumer, nous avons appliqué au Zarathoustra de Nietzsche la formule de Große modifiée en fonction des observations qui précèdent, nous limitant à un type particulier d'illocution qui est l'appel: appel=Indicateurs contextuels + présignaux + illocution indirecte (structures illocutoires complexes/mise en valeur) + illocution directe.

\section{La prédominance de l'«appel» dans le Zarathoustra}

Sans pouvoir entrer ici dans le détail, à la suite de l'analyse du Zarathoustra à l'aide de ces instruments, un constat s'impose d'emblée: l' " appel ", tel que nous avons tenté de le définir grâce aux apports de la pragmatique moderne, se manifeste ici sous toutes ses formes.

a) Sur le plan macrocommunicationnel (prioritaire si l'on hiérarchise les indices en fonction de leur effet) de l'illocution indirecte, nous mentionnerons d'abord les savoirs contextuels communément admis sur la philosophie de Nietzsche, lesquels savoirs sont partiellement à l'origine de l'hypothèse de départ qui nous a conduit à mener cette étude. Le vitalisme nietzschéen sollicite plus qu'il n'informe, le philosophe ne médite pas, il commande et ordonne, ce sont là des poncifs de l'exégèse nietzschéenne. Les professions de foi philosophiques - on pourrait dire métaphilosophiques - de Nietzsche lui-même, ne sont pas rares à cet égard. Qu'il nous suffise de mentionner ce passage particulièrement explicite de Jenseits von Gut und Böse: "Die eigentlichen Philosophen aber sind Befehlende und Gesetzgeber: sie sagen "so soll es sein!» [...] sie greifen mit schöpferischer Hand nach der Zukunft, und alles, was ist und war, wird ihnen dabei zum Mittel, zum Werkzeug, zum Hammer. Ihr "Erkennen" ist Schaffen, ihr Schaffen ist eine Gesetzgebung» (KGW VI.2.I49). Notons que ce contexte «martial», auquel on associe communément Nietzsche et sa philosophie, joue aussi par démarcage vis-à-vis d'un horizon d'attente lié au genre. C'est parce que la philosophie est traditionnellement associée de façon privilégiée à l'information que cette mise en exergue d'un faisceau d'indices contextuels associés à l'appel prend tout son relief.

b) En restreignant la notion de contexte à l'ensemble des œuvres et de la correspondance de Nietzsche (peut-être faut-il plutôt parler ici de cotexte), il convient de mentionner encore une fois (le phénomène est

50. Cf. Catherine Kerbrat-Orecchioni, L'implicite, Paris, Armand Colin, Linguistique, I986, p. I3 I (nous y reviendrons). 
surdéterminé) les multiples évocations de Zarathoustra comme œuvre, ou de Zarathoustra comme personnage, chronologiquement disséminées à travers une bonne partie des écrits, aussi bien en amont qu'en aval du Zarathoustra lui-même ( $c f$. supra). Ces évocations répétées, même si elles ne surviennent pas au sein de l'œuvre proprement dite, mais dans un corpus d'écrits satellites, relèvent de ce que nous avons appelé à la suite de Große le "facteur appel». "Zarathoustra ${ }^{51}$ ", constamment appelé et rappelé, crée une sorte de tonalité appellative diffuse autour de l'œuvre et du personnage. Le lecteur est en quelque sorte «mis en condition» pour recevoir dans toute sa force l'appel du Zarathoustra lui-même.

c) Dans le registre des "présignaux" (Große), on trouve bien évidemment le sous-titre souvent évoqué de l'ouvrage de Nietzsche: "Ein Buch für alle und für keinen». Ce sous-titre, qui ressemble à une sorte de «mode d'emploi » du livre à l'intention de ses utilisateurs, possède de ce simple fait une tonalité illocutoire inhabituelle. Il est assez rare en effet qu'un titre ou un sous-titre d'ouvrage insiste à ce point sur le cadre communicationnel, sur la destination et la réception potentielle de l'ouvrage par la communauté des lecteurs. Cela est d'autant plus vrai pour un texte présumé "philosophique ", qui aspire donc à l'universalité, et en principe aussi à une relative autonomie vis-à-vis de ses conditions de production/réception. Il y a donc dans ce sous-titre une mise en exergue de la communication qui est aussi frappante qu'elle est peu usuelle. Nietzsche se livre là, semble-t-il, à une véritable transgression des conventions paratextuelles, peut-être dans le but de "préparer » le destinataire à une appréhension non simplement cognitive, mais aussi communicationnelle de l'œuvre.

d) Dans ce même domaine des présignaux, on trouve, en dehors du soustitre, une série d'indices paratextuels, structurels et catégoriels à notre avis significatifs sur le plan illocutoire. Bien que le Zarathoustra soit un récit, une grande partie de l'œuvre est constituée par les "discours" de Zarathoustra explicitement désignés comme tels, pris «sur le vif» et rapportés tels quels au style direct, de façon pour ainsi dire "scénique ». Ce découpage scénique est encore mis en évidence par le jeu des intertitres qui précèdent chaque discours. C'est donc tout le principe de la communication théâtrale qui est ici mis à contribution: des personnages agissent constamment sur d'autres et les font réagir par leurs actes, mais surtout par leurs paroles (qui sont en l'occurrence aussi des actes). Le message philosophique ainsi théâtralisé ouvre potentiellement à l'" appel» sous toutes ses formes un champ beaucoup plus large et beaucoup plus riche que le message philosophique

51. Personnage ou œuvre. 
traditionnel, exposé par exemple sous forme de traité (dominante informative). À ce cadre général potentiellement propice à l'«appel » protéiforme s'ajoute le fait qu'une bonne partie des discours de Zarathoustra, s'il est permis de leur attribuer un genre, sont soit des harangues $^{52}$ adressées à un public que Zarathoustra apostrophe ("mes frères", "mes amis", etc.), soit des diatribes ${ }^{53}$. Or harangues et diatribes sont - par leur qualification générique même - en principe associées à l'appel.

e) Dans le registre des «illocutions indirectes", mais dans une sphère plus proche du microcommunicationnel, on trouve encore dans Zarathoustra quelques manifestations du «facteur appel», en particulier les évaluations axiologiques (lieben vs Hassen), en nombre incalculable dans l'œuvre. Les évaluations de Zarathoustra sont majoritairement subjectives et teintées d'affect (axiologico-affectifs), opposées à l'évaluation normative ${ }^{54}$.

Globalement, l'appel incite à la création de valeurs individuelles toujours renouvelées (dépassement des acquis). Évaluer (comme le fait

52. Le discours "Von den Fliegen des Marktes", par exemple, est une harangue explicitement tenue sur la place publique (Markt) et ponctuée par l'injonction plusieurs fois réitérée: «Fliehe, mein Freund, in deine Einsamkeit!» (KGW VI.I.6I-64).

53. Par exemple le chapitre "Von den Verächtern des Leibes». La diatribe est toujours partie prenante, partiale et partisane. Elle se situe incontestablement du côté de l'appel. Elle est en quelque sorte appel inversé: rejet (KGW VI.r.35-37).

54. Pour donner ponctuellement un exemple plus concret: on constate que Zarathoustra dans ses discours utilise fréquemment le verbe "nennen" et un peu moins souvent "heissen». Or les verbes «nennen»/ «heissen» sont dans beaucoup de cas des évaluatifs axiologiques qui permettent au locuteur d'évaluer une information en termes de vrai et de faux, cette évaluation vrai/faux étant étroitement liée à la personne à laquelle le verbe est utilisé: I) «Das nenne/ heisse ich ein Auto" $\rightarrow$ présupposé: «c'est vrai », 2) «Das nennst/heisst du ein Auto» $\rightarrow$ présupposé: «ce n'est pas vrai». Zarathoustra, lorsqu'il utilise le verbe "nennen", met donc à profit ces caractéristiques axiologiques pour stigmatiser subrepticement comme «faux» le point de vue de son interlocuteur ou, plus souvent, celui du tiers dont il s'entretient avec lui: «Bildung nennen sie ibren Diebstabl [...] sie erbrechen ibre Galle und nennen es Zeitung" (Vom neuen Götzen, KGW VI I.59), etc. Mais le plus intéressant est sans doute que Zarathoustra utilise aussi ponctuellement les évaluatifs axiologiques nennen/heissen à la première personne. Le verbe "nennen" est même le tout premier verbe utilisé par Zarathoustra dans son premier "discours", et c'est à la première personne qu'il l'utilise: "Drei Verwandlungen nenne ich euch des Geistes" (KGW VI I.25). Comme pour souligner cette "nomination ", cette dernière formule est d'ailleurs réitérée au prétérit en fin de chapitre. Zarathoustra utilise donc de façon stratégique et insistante l'évaluatif axiologique "nennen»: il évalue implicitement comme «vrais» ses propres propos, et cela d'entrée de jeu. On trouve dans la suite plusieurs autres occurrences de "nennen/heissen" à la première personne. Par l'intermédiaire de "nennen/heissen", le point de vue "vrai» de Zarathoustra s'oppose au point de vue "faux" des «autres » De façon à première vue assez curieuse pour un texte philosophique, le débat ne se situe manifestement pas sur le plan des «réalités ", mais sur le plan de l' "appellation » de ces réalités. On peut donc se poser la question: que suggère ce retournement impromptu du "vrai » en «faux", que rien ne semble motiver, sinon un simple «shifting» de personne: "j'appelle»= 


\section{Zarathoustra), c'est vouloir, mais c'est surtout vouloir que les autres veuillent et surpassent leur vouloir.}

f) Forte représentation aussi pour les figures de rhétorique, omniprésentes (pas uniquement la métaphore) dans l'œuvre, où elles focalisent sur certaines unités ou segments, créant ce qu'on a parfois appelé un « effet de présence ${ }^{55}$. Nous mentionnerons aussi, apparentés à la rhé-

"j'ai raison"; "tu/il appelle(s)» = «tu/il a tort »? Tout cela suggère bien évidemment en premier lieu la relativité de tout point de vue, relativité souvent thématisée chez Nietzsche: dans Zarathoustra, le chapitre Von tausend und einem Ziele (KGW VI I.70-72) est entièrement voué à cette thématique; il n'est donc guère étonnant d'y trouver plusieurs occurrences de "nennen/ heissen». De fait, le point de vue «vrai» n'est que celui d'un «je» qui l'évalue comme tel; le point de vue «faux" n'est que celui que ce «je" prête aux opposants que sont les «autres". La "vérité" n'est que l'expression de la force de celui qui - dans le combat vital - peut dire «j'appelle ceci le vrai ». Mais cette appellation-évaluation s'annule pour ainsi dire en se posant, puisqu'un autre peut dès cet instant la reprendre à son compte («il appelle ceci le vrai»), et que sa charge axiologique s'en trouve automatiquement retournée. On voit jusqu'à quel point l'évaluation, porteuse de signifiances philosophiques peut s'investir dans les caractéristiques microcommunicationnelles sciemment gérées.

55. Le discours Von den drei Verwandlungen est assez exemplaire à cet égard, il débute par une "personnification»: «um seinen Hochmuth Wehe zu tun?» (KGW VI.r.25) qui pourrait aussi être considérée comme une «métonymie» (l'orgueil auquel «on fait mal» est une qualité présumée de la personne à laquelle réfère le possessif «seiner»). Idem pour la formule jouxtant la précédente "um seiner Weisheit zu spotten?" (KGW VI.I.25). En dehors de ces figures d'ordre sémantique relevant de la substitution d'éléments, on trouve au début du passage même contenant les occurrences précédentes une «ellipse » soulignée par un double point: «Ist es nicht das: sich erniedrigen» (KGW VI.I.25). L'ellipse est ensuite répercutée à cinq reprises dans cinq formules réitérées à l'identique à deux lignes d'intervalle, et qui structurent elles-mêmes un "parallélisme » quintuple, mis en relief par autant d' "anaphores »: "Oder ist es das» [...] "Oder ist es das», usw. (KGW VI. I.25). Le premier membre de ce parallélisme contient une nouvelle figure basée sur le positionnement des éléments qui est la «dérivation » (proximité de termes remontant à la même racine lexicale): "um den Versuche zu versuchen" (KGW VI.r.25), peut-être encore souligné par l'allure pléonastique de la formule. Le deuxième membre du parallélisme contient manifestement une figure d'ordre "phonique/graphique» basée sur le cumul qui est l' "homéotéleute " (rapprochement de mots ayant les mêmes finales): "sich von Eicheln und Gras der Erkenntnis nähren und um der Wabrheit willen an der Seele Hunger leiden" (KGW VI.I.25). Même chose pour le cinquième membre du parallélisme: "Die lieben, die uns verachten, und dem Gespenste die Hand reichen, wenn es uns fürchten machen will» (KGW VI.I.25). Quant au cumul lexical et syntaxique, on a peut-être aussi, dans le quatrième membre du parallélisme, un exemple de "polysyndète " (suite de termes coordonnés): "wenn es das Wasser der Wahrheit ist, und kalte Frösche und heisse Kröten nicht von sich weisen» (KGW VI.r.25). Toujours dans le cadre de ce parallélisme, la figure de substitution sémantique la plus connue (et sans doute la plus répandue dans l'œuvre), à savoir la "métaphore", est représentée: "von Eicheln und Gras der Erkenntnis» (KGW VI.I.25) («Eichel und Gras» se substitue ici à «Baum» dans la formule consacrée «Baum der Erkenntnis")/ «asser der Wahrheit». Finalement, l'ensemble du parallélisme avec les alternatives qu'il propose (Oder...Oder, etc.) est repris par la formule synthétique "Alles diess Schwerste» (KGW VI.I.26), ce qui ressemble fort à ce que Perelman qualifie de "congérie ", à savoir une figure d'ordre sémantique reposant sur le cumul et qui énumère les parties d'un tout avant de les synthétiser. Les «répétitions" sont bien entendu dans tout le chapitre à la mesure des autres 
torique dans leurs effets, les procédés de mise en relief tels que la linéarisation expressive ${ }^{56}$, ou encore le double point (graphique), très répandu et dont l'effet le plus flagrant est la mise en relief des éléments situés en aval sur la chaîne graphique (cataphorisation), et de là, la focalisation sur «ce qui vient après ». En "ciblant » grâce à ces moyens (rhétoriques, graphiques ou syntaxiques) sur certaines séquences, on crée un effet de présence diffus qui contribue à créer une "ambiance appellative».

g) Quant à l'illocution directe qui fait intervenir les "voluntas signale», elle s'actualise soit dans des formules introductrices (Präsatze: «ich will/su sollst»), soit (majoritairement dans Zarathoustra) dans des formes de phrase (injonction, interrogation...), le tout induisant, à l'unisson de l'illocution indirecte véhiculée par l'ensemble des autres indices micro-et macrocommunicationnels, une forme d'appel extrêmement protéiforme, plus ou moins directif et/ou normatif selon le cas (nous réservons au chapitre suivant le traitement d'une illocution complexe liée au récit).

Or le simple constat de la prédominance de l'appel dans Zarathoustra est déjà en soi du plus haut intérêt. Définie au départ comme "quête" de vérité, la philosophie est "fonctionnellement" à la base du côté de l' «information » : objectivité, impartialité, sobriété sont sa devise. L'appel, quant à lui, se situerait plutôt du côté du mythe ou de la religion, face auxquels la philosophie s'est justement instituée en tant que telle par démarquage, mythe et religion étant en effet surtout injonction à croire. Si une telle simplification est permise, on pourrait dire que, pour le mythe ou la religion, ce à quoi l'on croit a relativement moins d'importance que le fait qu'il faille y croire. Or n'est ce pas là ce qui prime aussi dans le Zarathoustra? Le héros, qui se proclame "enseignant», pontifie, séduit et harangue beaucoup plus qu'il n'instruit (ce qui ne signifie aucunement qu'il n'instruit pas; la "critique des valeurs », par exemple, est liée à l'information). Dans le vocabu-

figures qui les impliquent (parallélisme, anaphore). Dans la suite immédiate, on trouve encore une prosopopée: "Aber der Geist des Löwen sagt, ich will" ", une personnification: "und also spricht der mächtigste aller Drachen"/«Also spricht der Drache», une «régression " (reprise de mots dans un ordre inverse à l'intérieur d'une phrase): "Aller Werth ward schon geschaffen, und aller geschaffene Werth", une "antithèse" intégrée à un parallélisme: "Neue Werthe schaffen - das vermag die Macht des Löwen noch nicht: aber Freiheit sich schaffen zu neuem Schaffen - das vermag die Macht des Löwen» (KGW VI.I.26). A la fin du chapitre, on relève encore, dans le registre du cumul lexical, une étonnante "énumération» de type asyndétique: "Unschuld ist das Kind und Vergessen, ein Neubeginnen, ein Spiel, ein aus sich rollendes Rad, eine erste Bewegung, ein heiliges Ja-sagen " (KGW VI.I.27).

56. Grammaire de l'allemand, formes et fonctions, Paris, Nathan Université, I986, p. 584-590. Le phénomène intervient dès la toute première phrase du premier "discours": "Drei Verwandlungen nenne ich euch des Geistes" (Von den drei Verwandlungen, KGW VI.I.25). 
laire de la rhétorique ancienne, on pourrait dire que son objectif relève plus du movere que du docere. En somme, contre toute attente, c'est l'appel et non l'information qui prévalent dans cette œuvre, laquelle se veut pourtant œuvre philosophique. Cette déviance, cette rupture même par rapport aux «conventions illocutoires» du genre, fait pour une bonne part l'originalité du Zarathoustra.

\section{L'appel comme parabole du vouloir}

Mais au-delà du constat de la «densité appellative» de Zarathoustra, nous avons aussi voulu — à travers l'analyse nuancée des différents types d'illocutions - montrer qu'un appel d'un type très particulier se construisait dans l'œuvre. Comme nous l'avons suggéré plus haut, certaines structures illocutoires complexes jouent dans et à travers le récit. Par le biais de ce que nous avons qualifié, à la suite de N. Everaert-Desmedt, de "contrat de séduction", elles contribuent à orienter l'"appel» véhiculé par le Zarathoustra dans une direction tout à fait inédite et absolument capitale en ce qui concerne les signifiances philosophiques.

De fait, en l'observant de près, on constate que le récit de Zarathoustra décrit un parcours événementiel et communicationnel original: Zarathoustra lance à ses auditoires (notamment sous forme de harangues) un «appel » aussi riche que varié. Plus précisément, cet appel se déploie en deux cycles formellement identiques, le premier englobant la première partie (parcours restreint), le second la totalité de l'œuvre (parcours global). Tout au long de la première partie, l'appel monte en puissance. Mais paradoxalement, parvenu à son point culminant, cet appel se retourne en son contraire: volontairement, Zarathoustra abandonne ses disciples et les encourage à l'abandonner également: "Allein gehe ich nun, meine Jünger. Auch ibr geht nun davon und allein! So will ich es» (Von der schenkenden Tugend, KGW VI.r.97). Et ce schéma se reproduit dans l'œuvre entière: Zarathoustra disparaît, délibérément là aussi, au moment précis où son public est conquis et où l'" appel » semble prêt à aboutir.

Or nous considérons pour notre part ces parcours paradoxaux comme une sorte de parabole du vouloir, une géniale tentative pour justement essayer d'échapper au paradoxe du vouloir, ce paradoxe qui comme Nietzsche lui-même le suggère à maintes reprises dans le Zarathoustra ${ }^{57}$ - se résume dans deux locutions verbales à la fois indissociables et incompatibles «ich will»/vs/ «du sollst». Qui "veut» impose son «vouloir », lequel dégénère fatalement en "pouvoir» pour celui qui l'exerce, et en "devoir » pour celui qui le subit. Pris dans ce cercle, le vouloir est condamné à se régénérer indéfiniment par la destruction de ses acquis. Ce vouloir, pour continuer à exister, s'inscrit inéluctablement dans

57. Le conflit philosophique du «ich will» (je veux) et du «du sollst» (tu dois) est annoncé dès le premier discours de cette première partie (Von den drei Verwandlungen). 
un schéma de création-destruction-recréation. Pour stimuler le vouloir de ses adeptes, Zarathoustra leur montre - à travers l'appel qu'il leur lance — son propre vouloir. Et il le leur montre bien sûr " en actes ", car le vouloir est forcément aussi irréductible à un quelconque «savoir». Ainsi, ce vouloir se potentialise, l'appel gagne l'auditoire. Mais du même coup — et c'est là encore une fois son paradoxe - ce vouloir se délite en pouvoir et en savoir. C'est donc là qu'il s'auto-détruit: en se niant, il se recrée et s'exacerbe. Or le vouloir, d'abord montré, puis nié, laisse un vide, un vide qui est précisément aussi un appel. Les disciples de Zarathoustra combleront ce vide par euxmêmes, car le vouloir est foncièrement intransitif. Quand un vouloir est pris pour "objet» par un autre, les deux vouloirs dégénèrent de concert. Le vouloir propre des «disciples» se substituera donc à celui du «maître». Et ainsi de suite dans un cycle sans fin. Ce sont les manifestations textuelles de ces phénomènes que nous tentons de saisir avec les notions $d$ ' "appel ipséiste » et d' «appel-dépassement ${ }^{58}$.

Certes, il faut - pour qu'une philosophie telle que celle de Nietzsche ait un sens - que le vouloir se transmette. Le vouloir nietzschéen est supraindividuel: vouloir, c'est vouloir que les autres veuillent. Mais vouloir que les autres veuillent signifie toujours «laisser vouloir» et jamais «faire vouloir». Autrement dit la transmission ne peut se faire que par "contagion». Cette transmission s'opère dans la continuité et non dans l'opposition des vouloirs: "Je veux et tu veux", mais surtout pas «je veux donc tu veux!». La suggestion est de mise, non l'injonction. Bien sûr Zarathoustra ordonne souvent, mais au final il retire ses ordres en se retirant lui-même. Globalement, le vouloir des autres n'est pas contraint, mais suscité. C'est donc dans ce contexte que nous avons retenu - entre autres - l'idée de contrat de séduction ( $c f$. supra). Par ses discours et ses actes Zarathoustra séduit ses «disciples» et fait naître en eux le désir, donc le vouloir. Élément capital: il n'y a dans ce procès aucune forme de coercition, aucune forme d'arraisonnement intellectuel (on parle souvent en ce sens d'arguments "contraignants »), psychologique (manipulation) ou à plus forte raison physique. À propos de l'" appel» lancé par Zarathoustra à ses auditoires, nous parlons également volontiers d' "empathie» («Einfühlung»). Vu du côté de Zarathoustra, cela pourrait aussi s'appeler du charisme!

Par ailleurs, puisqu'on ne pouvait conceptualiser le vouloir, il fallait le montrer «en exercice». Comme nous avons essayé de le prouver en d'autres lieux ${ }^{59}$, le vouloir, par sa nature même, ne peut s'inscrire dans le concept fixe

58. On ne peut vouloir qu'en niant ce qu'on a voulu et obtenu. Le vouloir est création continuée: il ne perdure qu'en faisant table rase de ses acquis et il prospère d'autant plus qu'il est apte à sacrifier - et cela une infinité de fois - ses réalisations les plus remarquables, ses biens les plus précieux et jusqu'à sa propre existence. Le second type d'appel, l'appel ipséiste, suggère qu'il n'est d'autre moyen de susciter le vouloir d'autrui que de le laisser vouloir par lui-même.

59. "L'écriture métaphorique dans le Zarathoustra de Nietzsche", Lectures d'une auvre, Also sprach Zarathoustra, Éditions du Temps, 2000, p. I89-205. 
et abstrait (Mummienbegriffe), il exige, par exemple, la métaphore (qui montre et transporte d'un lieu à l'autre), il exige, comme nous l'avons vu, la "métaphore énonciative» (cf. supra), et de la même façon, il demande enfin la parabole, seule susceptible de montrer ce vouloir "en marche», pris sur le vif dans un récit existentiel. Les discours et les parcours de Zarathoustra, la parabole qu'ils décrivent, étaient donc sans doute le seul moyen d'expression adapté au vouloir nietzschéen. Ce que Zarathoustra ne peut plus enseigner car ce n'est pas enseignable - le surhomme, l'éternel retour, la volonté de puissance qui sont en définitive des variantes de ce que nous qualifions synthétiquement de "vouloir» - il le vit sous nos yeux, il l'expérimente dans le trajet asymptotique qui le conduit, par la force de son vouloir, du néant à l'accomplissement et, par une surenchère de ce même vouloir, de l'accomplissement au néant. La signifiance philosophique véhiculée par l'appel est ici gravide de sens.

\section{Vouloir et «trope communicationnel»}

De là, pourquoi ne pas imaginer un schéma communicationnel à deux étages? Pourquoi ne pas lire derrière ces parcours fictionnels, un autre parcours communicationnel, réel celui-là, qui lierait Nietzsche à son lecteur? C'est l'hypothèse que nous avons faite, nous appuyant en cela sur la notion de «trope communicationnel» développée par C. Kerbrat-Orecchioni ( $c f$. supra). Plusieurs indices convergents semblent confirmer cette hypothèse, par exemple le fait que, dans Ecce homo, Nietzsche présente lui-même rétrospectivement son livre comme une "action" (qui lui serait donc imputable en propre): "Mein Begriff "dionysisch" wurde hier höchste Tat" (KGW VI.3.34I). Dans le même ouvrage, l'auteur évoque l'écriture du Zarathoustra comme un acte de dépassement, qui, si on le rapporte aux thèmes nietzschéens bien connus, pourrait être qualifié de «combat contre soi-même» ou encore de "performance vitale». En somme, même la production du Zarathoustra en tant qu'œuvre est présentée comme un acte de vouloir exacerbé. De là à penser que l'un des objectifs de Nietzsche était justement la transmission de ce vouloir, il n'y a qu'un pas.

Encore une fois, l'œuvre de Nietzsche ne peut se réduire au pur esthétisme; elle n'a pas non plus pour objectif unique de communiquer un savoir. Quelle autre vocation lui reste-t-il que justement celle de transmettre un vouloir? L'hypothèse du fonctionnement tropique et du schéma communicationnel à deux étages est, à notre avis, loin d'être aberrante. On l'a vu: par son retrait, Zarathoustra «laisse vouloir» son auditoire. C'était sans doute le seul moyen de contourner le paradoxe de la transmission du vouloir. Et de la même façon, Nietzsche «laisse vouloir» son lecteur. Il lui donne à lire une fiction, un récit existentiel qui est lui-même une parabole du vouloir (création-destruction-recréation). Mais il la lui donne à l'état "brut", sans consignes, sans clés de lecture. Afin de ne pas contrevenir à la nature même du vouloir, qui par définition ne s'impose pas, Nietzsche, par la neutralité 
philosophique qu'il affiche, laisse en fait le lecteur «vouloir» son œuvre. Ce lecteur peut adhérer ou ne pas adhérer à la parabole du vouloir que lui présente Nietzsche. L'option d'une lecture au "premier degré » reste ouverte; on peut fort bien appréhender le Zarathoustra comme une simple fiction, ignorer ses possibles implications dans la réalité communicationnelle. Mais on peut aussi faire une lecture au second degré, lire le Zarathoustra sur un mode tropique (c'est cela en fait le véritable objectif!), en "prenant pour soi » les propos de Zarathoustra. En tout état de cause, l'adhésion du lecteur à la parabole du vouloir, le fait qu'il la comprenne comme telle et en tire éventuellement des conséquences pour sa propre gouverne, doit être - de la part de ce lecteur - un acte de vouloir. Pour rester cohérent avec lui-même, Nietzsche ne pouvait s'accommoder d'un lecteur veule qui se contente de « digérer» des enseignements.

\section{Conclusion générale}

Le Zarathoustra dont on a souvent — au mépris des allégations de l'auteur lui-même - méconnu la portée philosophique, se révèle être au final une œuvre aussi riche (sinon plus) par sa forme que par ses contenus. Les signifiances discursives mises au jour nous révèlent une philosophie qui se questionne jusque dans les modalités de son "dire», interrogeant un discours millénaire, fréquemment aveugle à lui-même, dans lequel elle refuse de s'inscrire tacitement. La vérité est et reste tributaire du discours sur la vérité qui entend la révéler. Derrière le résultat inquestionné que pourvoit l'histoire épiphanique de la vérité et de l'être, Nietzsche sonde les sources, il ressaisit les genèses et les dynamiques qui agitent les profondeurs d'un discours dont on n'a voulu voir que la surface. C'est donc bien d'un renouveau qu'il faut parler, un renouveau qui engage, pourrait-on presque dire, l'onto- et la phylogenèse de l'œuvre, du discours générique et individuel qui la porte et lui donne forme et corps dans une langue. Plus qu'un «donné » abouti, Zarathoustra est montré comme un procès en marche, en prise constante avec ce dont il n'est ni plus ni moins qu'une manifestation fluctuante et adventice: la vie.

Mais le Zarathoustra comporte un autre versant, finalement complémentaire du premier: si la plupart des ouvrages philosophiques se veulent des sommes de "savoir", le Zarathoustra apparaît, lui, comme le "livre du vouloir". Le vouloir est partout, dans et autour de Zarathoustra. Il se reflète bien sûr dans la thématique (surhomme, éternel retour, volonté de puissance), dans la configuration interne de l'œuvre (récit en forme de parabole), dans les caractéristiques communicationnelles aussi bien en amont qu'en aval. Il se reflète dans la production, mais il se reflète aussi (et peut-être surtout) dans la réception de l'œuvre, œuvre qui est de part en part appel, appel à vouloir. L'exégèse nietzschéenne a beaucoup critiqué l'opacité théorique du Zarathoustra. Pourtant, Zarathoustra fait partie des œuvres qui ont fait couler le plus d'encre. Ce simple constat illustre peut-être mieux que tout le 
reste ce que Nietzsche entendait faire et ne pas faire: mobiliser les énergies plutôt que grossir les savoirs. Dans ce contexte la philosophie de Nietzsche apparaît comme une philosophie de l'action, une philosophie de l'"expérimentation ", à la fois poussée jusque dans ses ultimes conséquences, et étonnamment cohérente avec elle-même. Manifestation de la vie, cette philosophie a pour vocation de retourner nourrir la vie et ses mille expériences ${ }^{60}$, en suscitant et en potentialisant ce qui la nourrit elle-même: le vouloir.

\section{Bibliographie}

Ali-Bouacha, Magid. «De l'Ego à la classe des locuteurs»: lecture linguistique des Méditations", Langages n I 9 , L'analyse $d u$ discours philosophique, Paris, Larousse, septembre I995, p. 79-94.

Babich, Babette E. «On Nietzsche's concinnity» in Nietzsche-Studien, Internationales Jahrbuch für die Nietzsche-Forschung, Berlin, New York, Walter de Gruyter, Bd I9, I990, p. 65-80.

Benveniste, Emile. Problèmes de linguistique générale, Paris, Gallimard, II, 1974.

Blondel, Eric. Nietzsche, le corps et la culture, Paris, PUF, Philosophie d'aujourd'hui, I986.

Botet, Serge. Langue, langage et stratégies linguistiques chez Heidegger, Peter Lang, Bern, Berlin, Frankfurt/M, New York, Paris, Wien, 1997.

—. L'écriture métaphorique dans le Zarathoustra de Nietzsche, in Gilbert Merlio (ouvrage collectif coordonné par G. Merlio), Lectures d'une cuvre, Also sprach Zarathoustra, Paris, Éditions du Temps, 2000.

Brinker, Klaus. Linguistische Textanalyse, eine Einführung in Grundbegriffe und Methoden, Berlin, Erich Schmidt Verlag, Grundlagen der Germanistik, 4. Auflage, I997 (1985).

Cossuta, Frédéric. Éléments pour la lecture des textes philosophiques, Paris, Bordas, I989.

—. "Argumentation, ordre des raisons et mode d'exposition dans l'œuvre cartésienne», Descartes et l'argumentation philosophique, Paris, PUF, I996, p. III-I 85 .

Deleuze, Gilles. Nietzsche et la philosophie, Paris, Quadrige/PUF, 1962.

Ducrot, Oswald. Dire et ne pas dire, principes de sémantique linguistique. Paris, Hermann, Savoir, Sciences, I99I.

Eco, Umberto. Les limites de l'interprétation, Paris, Bernard Grasset, Biblio/Essais, I992.

Everaert-Desmedt, Nicole. Sémiotique du récit, Bruxelles, De Boeck Université, Prismes Méthodes 8, 1988.

Gauger, Hans-Martin. "Nietzsches Stil am Beispiel von Ecce homo", in Nietzsche Studien, internationales Jahrbuch für die Nietzsche-Forschung, Bd I3, I984, p. $332-355$.

60. En liaison avec le côté «expérimental» de la philosophie, l'Experimental-Philosophie, un auteur comme Volker Gerhardt écrit : "Eine Philosophie [...] die den Willen zur Macht nicht nur erkennt, sondern die anerkennt, dass sie selbst auch "nur " eine Äuberung des Willens zur Macht ist" (Volker Gerhardt, Pathos und Distanz, Studien zur Philosophie Friedrich Nietzsches, Stuttgart, Reclam, I988, p. 175). 
Gerhardt, Volker. Pathos und Distanz, Studien zur Philosophie Friedrich Nietzsches, Stuttgart, Reclam, I988.

Girard, René. La violence et le sacré, Paris, Grasset, 1987 (1972).

Granier, Jean. Le problème de la vérité dans la philosophie de Nietzsche, Paris, Seuil, I966.

Greimas, Algirdas Julien et Joseph Courtés. Dictionnaire raisonné de la théorie du langage, Paris, Hachette Université, I979.

Groddeck, Wolfram. "Die 'Neue Ausgabe' der 'Fröhlichen Wissenschaft'", Nietzsche-Studien, Internationales Jahrbuch für die Nietzsche-Forschung, Berlin, New York, Walter de Gruyter, Bd. 26, I997.

Große, Ernst Ulrich. Text und Kommunikation, Stuttgart, Berlin, Köln, Mainz, Verlag W. Kohlhammer.

Jeandillou, Jean-François. L'analyse textuelle, Paris, Armand Colin, 2007 (1997).

Jordan, Lothar. "Nietzsche Dekonstruktionist oder Konstruktivist", Nietzsche-Studien, Internationales Jabrbuch für die Nietzsche-Forschung, Bd 23, I994, p. 226-240.

Hagège, Claude. L'homme de paroles, contribution linguistique aux sciences humaines, Paris, Folio Essais, 1985.

Kerbrat-Orecchioni, Catherine. L'implicite, Paris, Armand Colin, Linguistique, I986.

Maingueneau, Dominique. "L'énonciation philosophique comme institution discursive», in Langages $\mathrm{n}^{\circ}$ II9, L'analyse $d u$ discours philosophique, Paris, Larousse, septembre 1995 .

Müller-Lauter, Wolfgang. "Nietzsches Lehre vom Willen zur Macht», in NietzscheStudien, Internationales Jahrbuch für die Nietzsche-Forschung, Bd 3, I974, p. 43 .

Nehamas, Alexander. "For Whom the Sun Shines", in Volkert Gerhardt (Hg.), Also sprach Zarathustra, Berlin, 2000, p. I65-190.

Platon, Théétète, Parménide. Traduction, notices et notes par Émile Chambry, Paris, Flammarion, 1967.

Rabatel, Alain. Une histoire du point de vue. Recherches textuelles $\mathrm{n}^{\circ}$ 2, publication du Centre d'études linguistiques des textes et des discours, Université de Metz, I997.

Sartre, Jean-Paul. L'Être et le Néant, essai d'ontologie phénoménologique, Paris, Gallimard, I943.

Schanen, François et Jean-Paul Confais. Grammaire de l'allemand, formes et fonctions, Paris, Nathan Université, I986.

Simon, Josef. "Ein Text wie Nietzsches Zarathustra », in Volker Gerhardt (Hg), Also sprach Zarathustra, Berlin, Akademie Verlag, Klassiker Auslegen, 2000, p. 225-256.

Stegmaier, Werner. "Anti-Lehren, Szene und Lehre», in Volker Gerhardt (Hg) Also sprach Zarathoustra, Berlin, Akademie Verlag, Klassiker auslegen, 2000, p. I9I-224. 